\title{
Wilson loops and chiral correlators on squashed spheres
}

\section{F. Fucito, ${ }^{a}$ J.F. Morales ${ }^{a}$ and R. Poghossian ${ }^{b}$}

${ }^{a}$ I.N.F.N - Sezione di Roma 2, and Dipartimento di Fisica, Università di Roma Tor Vergata, Via della Ricerca Scientifica, I-00133 Roma, Italy

${ }^{b}$ Yerevan Physics Institute, Alikhanian Br. 2, AM-0036 Yerevan, Armenia

E-mail: fucito@roma2.infn.it, morales@roma2.infn.it, poghos@yerphi.am

Abstract: We study chiral deformations of $\mathcal{N}=2$ and $\mathcal{N}=4$ supersymmetric gauge theories obtained by turning on $\tau_{J} \operatorname{tr} \Phi^{J}$ interactions with $\Phi$ the $\mathcal{N}=2$ superfield. Using localization, we compute the deformed gauge theory partition function $Z(\vec{\tau} \mid q)$ and the expectation value of circular Wilson loops $W$ on a squashed four-sphere. In the case of the deformed $\mathcal{N}=4$ theory, exact formulas for $Z$ and $W$ are derived in terms of an underlying $\mathrm{U}(N)$ interacting matrix model replacing the free Gaussian model describing the $\mathcal{N}=4$ theory. Using the AGT correspondence, the $\tau_{J}$-deformations are related to the insertions of commuting integrals of motion in the four-point CFT correlator and chiral correlators are expressed as $\tau$-derivatives of the gauge theory partition function on a finite $\Omega$-background. In the so called Nekrasov-Shatashvili limit, the entire ring of chiral relations is extracted from the $\epsilon$-deformed Seiberg-Witten curve. As a byproduct of our analysis we show that $\mathrm{SU}(2)$ gauge theories on rational $\Omega$-backgrounds are dual to CFT minimal models.

Keywords: Nonperturbative Effects, Wilson, 't Hooft and Polyakov loops, Supersymmetric gauge theory, Gauge-gravity correspondence

ARXIV EPRINT: 1507.05426 


\section{Contents}

1 Introduction and summary 1

2 The gauge partition function on $\mathbb{R}^{4} \quad 4$

2.1 The instanton moduli space and the equivariant charge 5

$\begin{array}{lll}2.2 & \text { The deformed gauge partition function } & 7\end{array}$

$\begin{array}{lll}2.3 \text { Chiral correlators } & 10\end{array}$

3 The gauge theory on $S^{4} \quad 10$

$\begin{array}{ll}3.1 \text { Wilson loops } & 11\end{array}$

$\begin{array}{lll}3.1 .1 & \text { Perturbative expansion } & 12\end{array}$

$\begin{array}{ll}3.2 \text { The } \mathcal{N}=4 \text { deformed theory } & 13\end{array}$

$\begin{array}{lll}3.3 & \text { Two point correlators }\left\langle\operatorname{tr} e^{\mathcal{C}} \operatorname{tr} \varphi^{J}\right\rangle & 16\end{array}$

4 AGT duality: chiral correlators vs integrals of motion $\quad 18$

$\begin{array}{lll}4.1 & \text { The CFT side } & 18\end{array}$

$\begin{array}{lll}\text { 4.1.1 The conformal field theory } & 18\end{array}$

$\begin{array}{ll}\text { 4.1.2 Integrals of motion } & 20\end{array}$

4.2 The gauge/CFT dictionary 21

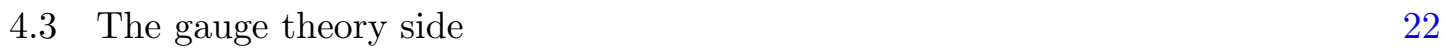

4.3.1 Chiral relations: $\epsilon_{1}, \epsilon_{2}$ finite 22

4.3.2 Deformed Seiberg-Witten curve: $h=\epsilon_{1} \epsilon_{2}=0 \quad 23$

5 AGT duals of minimal models $\quad 24$

5.1 The minimal models 24

5.2 Degenerated states vs. critical masses 26

5.2.1 The partition function and the four-points conformal blocks $\quad 27$

5.2.2 The gauge partition function on $S^{4}$ vs the four-point correlator 28

\section{Introduction and summary}

Wilson loops provide some of the most interesting observables to investigate in a gauge theory. Many of the intrinsic features of the gauge dynamics are encoded by Wilson loops of specific shapes. The quark-antiquark potential in a gauge theory with matter can be extracted from the asymptotic fall off of a Wilson loop made of two straight lines. The cusp anomalous dimension of the gauge theory can be extracted from the behaviour of the Wilson loop near a cusp. In the context of the AdS/CFT correspondence, the Wilson loop at strong coupling computes the minimal area on AdS of a string worldsheet ending 
on the loop at the AdS boundary and provides some of the most remarkable tests of the correspondence.

In theories with extended supersymmetry, the natural loops to consider are those preserving a fraction of the supersymmetry. The supersymmetric Wilson loop typically involves both the gauge field $A_{m}$ and its scalar superpartners $\varphi_{i}$

$$
W=\left\langle\operatorname{tr} e^{\mathcal{C}}\right\rangle \quad \text { with } \quad \mathcal{C}=\mathrm{i} \int\left(A_{m} \dot{x}^{m}+\varphi_{i} \dot{y}^{i}\right) d s
$$

Supersymmetry requires $|\dot{x}|=|\dot{y}|$. Many such solutions have been found preserving different fractions of the original supersymmetry [1-3].

In this paper we focus on circular Wilson loops for theories with $\mathcal{N}=2,4$ supersymmetry on a squashed $S^{4}$. Circular Wilson loops in $\mathcal{N}=4$ theory were first considered in [4] where it was conjectured that the perturbative series for $W$ at large $\mathrm{N}$ reduces to a simple counting of ladder diagrams. The counting of diagrams was extracted from a Gaussian matrix model [5]. An exact formula for $W$ was then proposed in [6] by an explicit evaluation of the Gaussian matrix model partition function to all orders in $1 / N$.

The conjectured formula for the circular Wilson loop in the $\mathcal{N}=4$ theory was later proved in [7] using localization. In that reference, the partition function of a general $\mathcal{N}=2$ gauge theory on a round sphere $S^{4}$ was shown to be given by the integral $\int d a|Z(a, \tau)|^{2}$ with $Z(a, \tau)$ the gauge partition function on $\mathbb{R}^{4}$. Here $Z$ is evaluated on an $\Omega$-background such that $\epsilon_{1}=\epsilon_{2}=\epsilon$ are finite, so the gauge theory lives effectively on a non-trivial gravitational background $[8,9]$. The function $Z(a, \tau)$ was previously computed in $[8,10-$ 14 by localization in the moduli space of instantons for theories with various gauge groups and matter content. On the other hand the expectation value of a circular Wilson loop on $S^{4}$ was shown to be given by the same integral with the insertion of the phase $\operatorname{tr} e^{\frac{2 \pi i a}{\epsilon}}$ inside the integral. Both $W$ and $Z$ are corrected by instantons and anti-instantons localized around the north and south poles of the sphere, whose contributions can be most easily accounted for if they come from the two different patches of which our space time is made of. The $\mathcal{N}=4$ Gaussian matrix model was then recovered from the case of $\mathcal{N}=2^{*}$ theory in the limit where the mass of the adjoint hypermultiplet is sent to zero.

It is natural to ask how these results are modified when the YM action is deformed or the spacetime is changed. An interesting spacetime to consider is that of a squashed sphere [15]. A motivation for considering four-dimensional gauge theories on squashed spheres comes from the AGT correspondence that relates the partition function of $\mathcal{N}=2$ supersymmetric theories to correlators in a dual two-dimensional CFT with the squeezing parameter $\epsilon_{1} / \epsilon_{2}$ parametrizing the central charge of the CFT [16, 17, 19]. In [18], it was proposed that the expectation value of a circular Wilson loop oriented along, let us say the first plane, on the squashed sphere is given by the insertion of the phase $\operatorname{tr} e^{\frac{2 \pi i a}{\epsilon_{1}}}$ in the partition function integral. In this paper we will prove this formula using localization and extend it to the case of a general $\mathcal{N}=2$ theory with prepotential $\mathcal{F}_{\text {class }}=\tau_{J} \operatorname{tr} \Phi^{J}$. This class of gauge theories were first introduced in [14] and the deformed partition function $Z(a, \vec{\tau})$ was computed in [13] (see also [21]) and related to the generating function of Gromov-Witten invariants for certain complex manifolds. 
In this paper we present a "physical derivation" of the deformed partition function based on localization and apply the results to the study of circular Wilson loops on squashed spheres. The crucial observation is that the circular Wilson loop $\mathcal{C}$ is nothing but the lowest component of the equivariant superfield $\mathcal{F}$ defining the field strength of a connection on the so called universal moduli space, the moduli space of instantons times the space time. Indeed in presence of an $\Omega$ background the zero mode solutions to the equations of motion get deformed and the lowest component of $\mathcal{F}$ reduces to $\tilde{\varphi}=\varphi+\delta_{\xi} x^{m} A_{m}$ with $\delta_{\xi} x^{m}$ a rotation of the spacetime coordinates along the Cartan of the Lorentz group. The same combination appears on the circular Wilson loop $\mathcal{C}=\frac{2 \pi \mathrm{i} n_{1}}{\epsilon_{1}} \tilde{\varphi}$, so the expectation value of the Wilson loop can be related to the $\Omega$-deformed version of the corresponding chiral correlator. Moreover using the fact that Wilson loops are allowed only on spheres with rational squeezing parameter $\epsilon_{1} / \epsilon_{2}$, an explicit evaluation of $\tilde{\varphi}$ shows that $\left\langle e^{\mathcal{C}}\right\rangle=\left\langle e^{\frac{2 \pi i_{1}}{\epsilon_{1}} a}\right\rangle$, so all instanton corrections to $\mathcal{C}$ (but not to the correlator) cancel after exponentiation! The same cancellation was observed in [7] for the case of the round sphere. Similarly $\operatorname{tr} \Phi^{J}$ interactions can be related to the higher component of $\operatorname{tr} \mathcal{F}^{J}$, and their expectation values are again given by the deformed chiral correlators up to some super-volume factors.

The case of the deformed $\mathcal{N}=4$ theory is particularly interesting. $\tau_{J}$-interactions break supersymmetry down to $\mathcal{N}=2$ but surprisingly the resulting theory is far more simpler than any other $\mathcal{N}=2$ theory one can think of. For instance, as we will see, instanton contributions to the partition function and circular Wilson loops can be shown to cancel in the deformed theory more in the same way than in the maximally supersymmetric theory. As a consequence the gauge partition function and the expectation values of circular Wilson loops are described by an effective interacting matrix model. More precisely, the Wilson loop is given by the integral

$$
\left\langle\operatorname{tr} e^{\mathcal{C}}\right\rangle=\frac{1}{Z} \int_{\mathbb{R}} d^{N} a \Delta(a) \operatorname{tr} e^{\frac{2 \pi a}{\epsilon_{1}}} e^{-N V(\vec{\tau}, a)}
$$

with $\Delta(a)$ the Vandermonde $\mathrm{U}(N)$ determinant and $V(\vec{\tau}, a)$ a potential codifying the $\tau_{J^{-}}$ interactions. The matrix model integral effectively counts the number of Feynman diagrams with propagators ending on the Wilson loop, and internal J-point vertices weighted by $\tau_{J}$. The case with only quartic interactions will be worked out in full details. A formula for $W$ at large $N$ but exact in $g_{Y M}$ will be derived in this case. This formula provides a simple prediction $S=\ln W \sim \sqrt{\lambda_{\text {eff }}}$ for the minimal area of a string worldsheet on the gravity dual theory as a function of a $\tau_{4}$-dependent effective t'Hooft coupling $\lambda_{\text {eff }}$. As a bonus, in this case, it will also be possible to find the correlators of the Wilson loop with $\operatorname{tr} \Phi^{2}$ and $\operatorname{tr} \Phi^{4}$ and check our results against those of [20]. ${ }^{1}$

Exploiting the AGT correspondence between 4d gauge theories and 2d CFT's, the chiral correlators $\left\langle\operatorname{tr} \tilde{\varphi}^{J}\right\rangle$ in the gauge theory can be related to insertions of the integrals of motions $I_{J}$ into the four-point CFT correlator computing the gauge theory partition function $[21,23]$. The computation of such deformed correlators is feasible and it leads to relations which are the analogue of the chiral ring relations for $\mathcal{N}=2$ gauge theories [24]

\footnotetext{
${ }^{1}$ We thank the referee of the paper to point out this reference to us.
} 
for a finite $\Omega$ background. We carry out the explicit computation in the $\mathrm{SU}(2)$ theory with four fundamentals for the simplest chiral correlators and show the match with the dual correlators in the Liouville theory. We also consider higher point correlators in the so called Nekrasov-Shatshvili limit in which one of the two deformation parameters of the $\Omega$ background is sent to zero. In this limit an $\epsilon$-deformed version of the Seiberg-Witten type curve is available $[25,26]$ from which we extract the full set of chiral ring relations.

Finally, focusing on rational $\Omega$-backgrounds (those admitting Wilson loops) we propose a duality between certain $\mathrm{SU}(2)$ gauge theories with four fundamental hypermultiplets of critical masses and minimal models (see [27-31] for previous studies of this duality). These critical gauge theories fall into a general class of models studied in [32] where the entire instanton contributions can be explicitly resummed in terms of hypergeometric functions. The gauge duals of all 4-point correlators of the Ising models are identified and the gauge partition functions are matched against the dual CFT correlators.

The paper is organised as follows: in section 2 we review the derivation of the deformed partition function for $\mathcal{N}=2$ gauge theories with adjoint or fundamental matter on $\mathbb{R}^{4}$. In section 3 we consider the gauge theory on the squashed sphere $S^{4}$. We derive a localization formula for the Wilson loop in the deformed theory and discuss in details the case of the $\mathcal{N}=4$ theory deformed by a quartic $\tau_{4}$-interaction. In section 4 we describe the AGT duality between the chiral correlators in the $\mathrm{SU}(2)$ gauge theory and the insertions of integrals of motion in the Liouville four-point function. We show that chiral correlators in the gauge theory can be written in terms of derivatives of the gauge theory partition function and show how chiral ring relations can be extracted from the $\epsilon$-deformed SeibergWitten curve in the limit in which one of the two parameters of the $\Omega$-background is sent to zero. In section 5, we describe the duality between $\mathrm{SU}(2)$ gauge theories with critical masses and rational $\Omega$-backgrounds and CFT minimal models.

\section{The gauge partition function on $\mathbb{R}^{4}$}

The action of a general $\mathcal{N}=2$ supersymmetric gauge theory on $\mathbb{R}^{4}$ is specified by a single holomorphic function, the prepotential, $\mathcal{F}_{\text {class }}(\Phi)$ of the $\mathcal{N}=2$ vector multiplet superfield $\Phi$. Explicitly

$$
S_{\text {class }}=\left[\frac{1}{4 \pi^{2}} \int d^{4} x d^{4} \theta \mathcal{F}_{\text {class }}(\Phi)+\text { h.c. }\right]+\ldots
$$

with $\left(x_{m}, \theta_{\alpha \dot{a}}\right)$ denoting the super-space coordinates and the lower dots denoting couplings to hypermultiplets. We are interested in the Coulomb branch of the gauge theory where the scalar $\varphi$ in the vector multiplet $\Phi$ has a non-trivial vacuum expectation value. In this branch, only the vector multiplet prepotential is corrected by quantum effects. We take the classical prepotential of the general form

$$
\mathcal{F}_{\text {class }}(\Phi)=\sum_{J=2}^{p} \frac{2 \pi \mathrm{i} \tau_{J}}{J !} \operatorname{tr} \Phi^{J}
$$

for some integer $p$ and

$$
\Phi=\varphi+\lambda_{m} \theta^{m}+\frac{1}{2} F_{m n} \theta^{m} \theta^{n}+\ldots
$$


Here and in the rest of the paper we used the "twisted" fermionic variables $\theta^{m}=\frac{1}{2} \sigma^{m \alpha \dot{a}} \theta_{\alpha \dot{a}}$ obtained by identifying internal $\dot{a}$ and Lorenz $\dot{\alpha}$ spinors indices. The gauge theory following from (2.2) can be seen as a deformation of the standard renormalizable gauge theory where the gauge coupling is replaced by a function of the scalar field $\varphi$ and the super symmetrically related couplings $F \lambda^{2}$ and $\lambda^{4}$ are included. The standard theory with prepotential $\mathcal{F}_{\text {class }}(\Phi)=\pi \mathrm{i} \tau \operatorname{tr} \Phi^{2}$ is recovered after setting $\tau_{J>2}$ to zero.

We will refer to the partition function $Z(\vec{\tau}, q)$ as the "deformed partition function". There are two main contributions to the partition function $Z_{\text {one-loop }}$ and $Z_{\text {tree+inst }}(\vec{\tau}, q)$ coming from the fluctuations of the gauge theory fields around the trivial and the instanton vacua. We notice that only the latter one depends on the couplings $\tau_{J}$ since $Z_{\text {one-loop }}$ is given by a one-loop vacuum amplitude. On the other hand $Z_{\text {tree+inst }}(\vec{\tau}, q)$ can be written in terms of an integral over the instanton moduli space that can be computed with the help of localization. We start by reviewing the construction of the instanton moduli space and introducing the basic ingredient for localization: the equivariant supercharge $Q_{\xi}$ and the equivariant vector superfield $\mathcal{F}$. For concreteness we focus on conformal gauge theories with gauge group $\mathrm{U}(N)$ and fundamental or adjoint matter.

\subsection{The instanton moduli space and the equivariant charge}

The moduli space of instanton solutions can be packed into a bosonic-fermionic pair of $[N+2 k] \times 2 k$ matrices $\Delta_{0}, \mathcal{M}$ characterizing the instanton gauge connection and the gaugino zero modes. The instanton gauge connection is built out of the ADHM matrix $\Delta=\Delta_{0}+x_{m} b_{m}$, with $b_{m}$ a constant and moduli independent matrix. The matrices $\Delta, \mathcal{M}$ satisfy the super-ADHM constraints

$$
\bar{\Delta} \Delta \sim \mathbb{1}_{[2] \times[2]} \quad \bar{\Delta} \mathcal{M}+\overline{\mathcal{M}} \Delta=0
$$

The ADHM constraints (2.4) together with the obvious $\mathrm{U}(k)$ symmetry of the construction lead to $4 k^{2}$ bosonic and fermionic relations for the $4 k N+4 k^{2}$ bosonic and fermionic degrees of freedom in $\Delta_{0}, \mathcal{M}$ leaving a $4 k N$-dimensional superspace $\mathfrak{M}_{k, N}$. Fundamental hypermultiplets contribute an extra $\left[k \times N_{f}\right]$ matrix $\mathcal{K}$ of fermionic moduli that can be conveniently paired with the auxiliary field $h$ in the same representation. The instanton connection can be written in terms of the $[\mathrm{N}+2 \mathrm{k}] \times[\mathrm{N}]$ matrix $U$ defined by

$$
\Delta U=\bar{U} \Delta=0 \quad \bar{U} U=\mathbb{1}
$$

and reads

$$
A_{m}=\bar{U} \partial_{m} U
$$

To localize the integral one first introduces the equivariant charge on $\mathfrak{M} \times \mathbb{C}^{2}$,

$$
Q_{\xi}=Q+d+i_{\xi}
$$

with $d=d x^{m} \partial_{m}$ the exterior differential on $\mathbb{C}^{2}, Q$ a supersymmetry component acting on the moduli space via

$$
\begin{array}{rlrl}
Q \Delta & =\mathcal{M} & Q \mathcal{M} & =0 \\
Q \mathcal{K} & =h & Q h & =0
\end{array}
$$


and $i_{\xi}$ a contraction defined by

$$
i_{\xi} d x^{m}=\delta_{\xi} x^{m} \quad i_{\xi} d \mathfrak{M}_{i}=\delta_{\xi} \mathfrak{M}_{i}
$$

with $\delta_{\xi}$ an element of the symmetry group $\mathrm{SO}(4) \times \mathrm{U}(k) \times \mathrm{U}(N) \times \mathrm{U}\left(N_{f}\right)$ rotating moduli and spacetime coordinates. The combined action can then be written as

$$
\begin{array}{llrl}
Q_{\xi} \Delta & =\mathcal{M}+d \Delta & Q_{\xi}^{2} \Delta & =\delta_{\xi} \Delta \\
Q_{\xi} \mathcal{K} & =h & Q_{\xi}^{2} \mathcal{K} & =\delta_{\xi} \mathcal{K}
\end{array}
$$

We notice that $Q_{\xi}^{2}=\delta_{\xi}$, so $Q_{\xi}$ is nilpotent at the fixed points of $\delta_{\xi}$. The charge $Q_{\xi}$ can be thought of as an equivariant version of the supersymmetry charge. The second ingredient for localization, is the construction of an equivariant field $\mathcal{F}$ defined by

$$
\mathcal{F}=Q_{\xi}\left(\bar{U} Q_{\xi} U\right)+\left[\bar{U} Q_{\xi} U, \bar{U} Q_{\xi} U\right]=\tilde{\varphi}+\lambda+F
$$

with

$$
\begin{aligned}
\tilde{\varphi} & =\bar{U} \delta_{\xi} U+Q \bar{U} Q U+[\bar{U} Q U, \bar{U} Q U] \\
\lambda & =\nabla(\bar{U} Q U) \\
F & =\nabla(\bar{U} d U)
\end{aligned}
$$

the 0,1 and 2-form parts of $\mathcal{F}$ on $\mathbb{C}^{2}$ and $\nabla=d+[A,$.$] . We notice that at finite \epsilon$ 's the field $\tilde{\varphi}$ involves a non-trivial linear combination of the scalar $\varphi$ and the vector field $A_{m}$ components. Indeed, recalling that $\delta_{\xi}$ rotates both the instanton moduli and the spacetime variables $x^{m}$ one can write [33]

$$
\tilde{\varphi}=\bar{U} \delta_{\xi}^{\bmod } U+Q \bar{U} Q U+[\bar{U} Q U, \bar{U} Q U]+\delta_{\xi} x^{m} A_{m}=\varphi+\delta_{\xi} x^{m} A_{m}
$$

In the limit $\epsilon_{\ell}$ to zero, $\delta_{\xi} x^{m} \rightarrow 0, \tilde{\varphi}$ reduces to $\varphi$ and the equivariant correlator computes the scalar correlators of the gauge theory on $\mathbb{R}^{4}$. In this paper, we are interested on gauge theories living on a non-trivial $\Omega$-background so we keep $\epsilon_{1}, \epsilon_{2}$ finite and the supersymmetric operators are built out of $\tilde{\varphi}$ rather than $\varphi$.

It is important to notice that $\mathcal{F}$ coincides with the $\mathcal{N}=2$ vector superfield $\Phi(2.3)$ after replacing $\theta^{m}$ by $d x^{m}$ and evaluating the fields on the instanton background. Indeed, one can check that higher $\theta$-terms in (2.3) cancel on the instanton solution. The field $\mathcal{F}$ can then be viewed as the equivariant version of the $\mathcal{N}=2$ superfield $\Phi$. Moreover

$$
Q_{\xi} \mathcal{F}=\left(\delta_{\xi}+\delta_{\text {gauge }}\right)\left(\bar{U} Q_{\xi} U\right)
$$

i.e. $\mathcal{F}$ is $Q_{\xi}$-invariant up to a symmetry rotation. This implies that any invariant function made out of $\mathcal{F}$ is $Q_{\xi}$-closed. In particular the Yang-Mills action (2.2) in the instanton background can be written in the explicit $Q_{\xi}$-invariant form

$$
S_{\text {inst }}(\vec{\tau})=\sum_{J=2}^{p} \frac{\mathrm{i} \tau_{J}}{2 \pi J !} \int_{\mathbb{C}^{2}} \operatorname{tr} \mathcal{F}^{J}
$$




\subsection{The deformed gauge partition function}

The gauge partition function on $\mathbb{R}^{4}$ can be written as the product of the one-loop, tree level and instanton contributions

$$
Z(\vec{\tau})=Z_{\text {one-loop }} Z_{\text {inst+tree }}(\vec{\tau})
$$

The one-loop partition function $Z_{\text {one-loop }}$ is given by

$$
Z_{\text {one-loop }}=Z_{\text {one-loop }}^{\text {gauge }} Z_{\text {one-loop }}^{\text {matter }}
$$

with $[17,32]$

$$
\begin{aligned}
Z_{\text {one-loop }}^{\text {gauge }} & =\prod_{u<v}^{N} \Gamma_{2}\left(a_{u v}\right)^{-1} \Gamma_{2}\left(a_{u v}+\epsilon\right)^{-1} \\
Z_{\text {one-loop }}^{\text {matter }} & = \begin{cases}\prod_{u<v}^{N} \Gamma_{2}\left(a_{u v}-m\right) \Gamma_{2}\left(a_{u v}+m+\epsilon\right) & \text { adj. } \\
\prod_{u, v=1}^{N} \Gamma_{2}\left(a_{v}-\bar{m}_{u}\right) \Gamma_{2}\left(a_{u}-m_{v+N}+\epsilon\right) & \text { fund. }\end{cases}
\end{aligned}
$$

Here $\Gamma_{2}(x)$ is the Barnes double gamma function ${ }^{2}$ and $\epsilon=\epsilon_{1}+\epsilon_{2}$.

The instanton partition function is defined by the moduli space integral

$$
Z_{\text {inst+tree }}(\tau)=\sum_{k=0}^{\infty} \int d \mathfrak{M}_{k, N} e^{-S_{\text {inst }}(\vec{\tau})}
$$

with the integral running over the instanton moduli space for a given $k$. The action $S_{\text {inst }}$ and the integral over the instanton moduli spaces can be evaluated with the help of localization. According to the localization theorem, given an equivariant derivative $Q_{\xi}$, and an action $\delta_{\xi}=Q_{\xi}^{2}$ on a space $M$, the integral of any $Q_{\xi}$-closed equivariant form $\alpha$ is given by the localization formula [12]

$$
\int_{M} \alpha=(-2 \pi)^{\frac{D}{2}} \sum_{x_{0}} \frac{\alpha_{0}}{\operatorname{det} \delta_{\xi}}
$$

with $D$ the complex dimension of the space $M, x_{0}$ labelling the fixed points of $\delta_{\xi}$ and $\alpha_{0}$ the 0 -form part, with respect to the equivariant $Q$-grading, of $\alpha$. To evaluate $S_{\text {inst }}(\vec{\tau})$, we take $M=\mathbb{C}^{2}, \alpha=\sum_{J=2}^{\infty} \frac{\tau_{J}}{2 \pi J !} \operatorname{tr} \mathcal{F}^{J}$ and $\delta_{\xi}$ a rotation in the Cartan of the $\mathrm{SO}(4)$ Lorentz group acting as $z_{\ell} \rightarrow e^{i \epsilon_{\ell}} z_{\ell}$ on the complex coordinates of $\mathbb{C}^{2}$. The unique fixed point of this action is the origin and the localization formula leads to

$$
S_{\text {inst }}(\vec{\tau})=\sum_{J=2}^{p} \frac{\mathrm{i} \tau_{J}}{2 \pi J !} \int_{\mathbb{C}^{2}} \operatorname{tr} \mathcal{F}^{J}=\frac{2 \pi \mathrm{i}}{\epsilon_{1} \epsilon_{2}} \sum_{J=2}^{p} \frac{\tau_{J}}{J !} \operatorname{tr} \tilde{\varphi}(0)^{J}
$$

To perform the integral over the instanton moduli space we take $\alpha=e^{-S_{\text {inst }}(\vec{\tau})}$ and $\delta_{\xi}$ an element of the Cartan subgroup of the symmetry group $\mathrm{U}(N) \times \mathrm{U}\left(N_{f}\right) \times \mathrm{U}(k) \times \mathrm{SO}(4)$.

\footnotetext{
${ }^{2}$ The Barnes double gamma function can be thought of as a regularization of the infinite product (for $\left.\epsilon_{1}>0, \epsilon_{2}>0\right)$

$$
\Gamma_{2}(x)=\prod_{i, j=0}^{\infty}\left(\frac{\Lambda}{x+i \epsilon_{1}+j \epsilon_{2}}\right) .
$$


We parametrize the Cartan element by $a_{u}, m_{i}, \chi_{I}, \epsilon_{1,2}$. More precisely, $a_{u}$ parametrizes the eigenvalues of the vacuum expectation value matrix $\langle\varphi\rangle, m_{i}$ the masses of the fundamental or adjoint fields, $\chi_{I}$ the Cartan of $\mathrm{U}(\mathrm{k})$ and $\epsilon_{1,2}$ are Lorentz breaking parameters that deform the $\mathbb{R}^{4}$ spacetime geometry. For a gauge theory on flat space $\epsilon_{1,2}$ should be sent to zero at the end of the computation. For finite $\epsilon$ 's the integral describes the partition function on a non-trivial gravitational background, the so called $\Omega$-bakcground. Fixed points of $\delta_{\xi}$ are again isolated and are in one-to-one correspondence with N-tuples of Young tableaux $Y=\left(Y_{1}, \ldots Y_{N}\right)$ with a total number of $k$ boxes. The Young tableaux $Y$ specify the $\mathrm{U}(\mathrm{k})$ Cartan elements $\chi_{I}$. Explicitly

$$
\chi_{(i, j)}=a_{u}+(i-1) \epsilon_{1}+(j-1) \epsilon_{2} \quad(i, j) \in Y_{u}
$$

In our conventions $\epsilon_{1}, \epsilon_{2}$ are pure real numbers. From (2.21) one finds for the moduli space integral

$$
Z_{\text {inst }+ \text { tree }}(\tau)=\sum_{Y} Z_{Y} e^{-S_{Y}(\vec{\tau})}
$$

with $Z_{Y}$ the inverse determinant of $\delta_{\xi}$ and $S_{Y}(\vec{\tau})$ the 0-form of the instanton action $(2.22)$ evaluated at the fixed point. For $Z_{Y}$ one finds $[11,12,32]$

$$
Z_{Y}=\left.\frac{1}{\operatorname{det} \delta_{\xi}}\right|_{Y}=Z_{Y}^{\text {gauge }} Z_{Y}^{\text {matter }}
$$

with

$$
\begin{aligned}
Z_{Y}^{\text {gauge }} & =\prod_{u, v}^{N} Z_{Y_{u}, Y_{v}}\left(a_{u v}\right)^{-1} \\
Z_{Y}^{\text {matter }} & = \begin{cases}\prod_{u, v}^{N} Z_{Y_{u}, Y_{v}}\left(a_{u v}+m\right) & \text { adjoint } \\
\prod_{u, v=1}^{N} Z_{\emptyset, Y_{v}}\left(\bar{m}_{u}-a_{v}\right) Z_{Y_{u}, \emptyset}\left(a_{u}-m_{v+N}\right) & \text { fund }\end{cases}
\end{aligned}
$$

and

$$
\begin{aligned}
Z_{Y_{u}, Y_{v}}(x)= & \prod_{(i, j) \in Y_{u}}\left(x-\epsilon_{1}\left(k_{v j}-i\right)+\epsilon_{2}\left(1+\tilde{k}_{u i}-j\right)\right) \\
& \times \prod_{(i, j) \in Y_{v}}\left(x+\epsilon_{1}\left(1+k_{u j}-i\right)-\epsilon_{2}\left(\tilde{k}_{v i}-j\right)\right)
\end{aligned}
$$

Here $(i, j)$ run over rows and columns respectively of the given Young tableaux, $\left\{k_{u j}\right\}$ and $\left\{\tilde{k}_{u i}\right\}$ are positive integers giving the length of the rows and columns respectively of the tableau $Y_{u}$. We remark that (2.18) and (2.26) are not symmetric under the exchange of fundamental $\bar{m}_{i}$ and anti-fundamental masses $\bar{m}_{j}$ but a totally symmetric form under the exchange $\bar{m}_{i} \leftrightarrow \bar{m}_{j}$ can be obtained by replacing

$$
m_{u+N} \rightarrow \bar{m}_{u+N}+\epsilon
$$

Finally, the contribution of the deformed Yang-Mill action at the fixed point $Y$ reduces to

$$
S_{Y}(\vec{\tau})=\left.\frac{2 \pi \mathrm{i}}{\epsilon_{1} \epsilon_{2}} \sum_{J=2}^{p} \frac{\tau_{J}}{J !} \operatorname{tr} \tilde{\varphi}_{0}^{J}\right|_{Y}=\frac{2 \pi \mathrm{i}}{\epsilon_{1} \epsilon_{2}} \sum_{J=2}^{p} \frac{\tau_{J}}{J !} \mathcal{O}_{J, Y}
$$


with $\tilde{\varphi}_{0}=\bar{U} \delta_{\xi} U$ the zero form of the equivariant superfield $\mathcal{F}$ and $\mathcal{O}_{J, Y}$ the chiral primary operator evaluated at the fixed point. To evaluate $\mathcal{O}_{J, Y}$, one first notices that for a $\Delta$ specified by the Young tableaux data $Y$ one can find infinitely many solutions $U^{(k, l)}$ to the defining equation $\bar{\Delta} U=0$. The $\delta_{\xi}$-eigenvalue of $U^{(k, l)}$ is given by $\chi_{(k, l)}=a_{u}+(k-1) \epsilon_{1}+$ $\epsilon_{2}(l-1)$ with $(k, l) \notin Y_{u}$ [33]. Summing over $(k, l)$ one finds for the generating function of the chiral operators $[14,33]$.

$$
\begin{aligned}
\left.\operatorname{tr} e^{z \tilde{\varphi}_{0}}\right|_{Y} & =\mathcal{V} \sum_{u=1}^{N} \sum_{(k, l) \notin Y_{u}} e^{z \chi_{(k, l)}}=\mathcal{V} \sum_{u=1}^{N}\left(\sum_{k, l} e^{z \chi_{(k, l)}}-\sum_{k, l \in Y_{u}} e^{z \chi_{(k, l)}}\right) \\
& =\sum_{u}\left(e^{z a_{u}}-\left(1-e^{z \epsilon_{1}}\right)\left(1-e^{z \epsilon_{2}}\right) \sum_{(i, j) \in Y_{u}} e^{z \chi_{(i, j)}}\right)
\end{aligned}
$$

where we have included the normalization factor $\mathcal{V}=\left(1-e^{z \epsilon_{1}}\right)\left(1-e^{z \epsilon_{2}}\right)$ in order to reproduce the classical result $\left\langle e^{z \varphi}\right\rangle_{\text {class }}=\operatorname{tr} e^{z a}$. Expanding both sides of (2.30) in powers of $z$ one finds

$$
\mathcal{O}_{J, Y}=\sum_{u}\left(a_{u}^{J}-\sum_{(i, j) \in Y_{u}}\left[\chi_{(i, j)}^{J}+\left(\chi_{(i, j)}+\epsilon\right)^{J}-\left(\chi_{(i, j)}+\epsilon_{1}\right)^{J}-\left(\chi_{(i, j)}+\epsilon_{2}\right)^{J}\right]\right)
$$

For the first few values of $J$ one finds

$$
\begin{aligned}
& \mathcal{O}_{2, Y}=\operatorname{tr} a^{2}-2 k \epsilon_{1} \epsilon_{2} \\
& \mathcal{O}_{3, Y}=\operatorname{tr} a^{3}-3 \epsilon_{1} \epsilon_{2} \sum_{u=1}^{N} \sum_{(i, j) \in Y_{u}}\left(\epsilon_{1}+\epsilon_{2}+2 \chi_{(i, j)}\right) \\
& \mathcal{O}_{4, Y}=\operatorname{tr} a^{4}-4 \epsilon_{1} \epsilon_{2} \sum_{u=1}^{N} \sum_{(i, j) \in Y_{u}}\left(2 \epsilon_{1}^{2}+3 \epsilon_{1} \epsilon_{2}+2 \epsilon_{2}^{2}+6 \epsilon \chi_{(i, j)}+6 \chi_{(i, j)}^{2}\right)
\end{aligned}
$$

The deformed partition functions can then be written as

$$
Z_{\text {inst+tree }}(\vec{\tau})=\sum_{Y} Z_{Y}(\vec{\tau})=\sum_{Y} Z_{Y} \exp \left(-\frac{2 \pi \mathrm{i}}{\epsilon_{1} \epsilon_{2}} \sum_{J=2}^{p} \frac{\tau_{J}}{J !} \mathcal{O}_{J, Y}\right)
$$

with $\mathcal{O}_{J, Y}$ given by $(2.31)$. The gauge prepotential $\mathcal{F}_{\text {eff }}$ is then identified with the free energy associated to the deformed instanton partition function

$$
\mathcal{F}_{\text {eff }}(\vec{\tau})=-\epsilon_{1} \epsilon_{2} \ln Z(\vec{\tau})
$$

We notice that the deformed partition function (2.16) and the prepotential (2.34) reduce to the partition function and prepotential of the undeformed $\mathcal{N}=2$ gauge theory if we take $\tau_{J>2}=0$. Indeed using (2.32) for $J=2$ one finds

$$
Z\left(\tau_{J}=\tau \delta_{J, 2}\right)=e^{-\frac{\pi \mathrm{i} \tau \sum_{u} a_{u}^{2}}{\epsilon_{1} \epsilon_{2}}} Z_{\text {oneloop }} \sum_{Y} Z_{Y} q^{|Y|}
$$

with $q=e^{2 \pi \mathrm{i} \tau}$ and $Z_{\text {tree }}=e^{-\frac{\pi \mathrm{i} \tau \sum_{u} a_{u}^{2}}{\epsilon_{1} \epsilon_{2}}}$ the tree level contribution to the partition function and $Z_{\text {inst }}=\sum_{Y} Z_{Y} q^{|Y|}$ the instanton part. 


\subsection{Chiral correlators}

In presence of a non-trivial $\Omega$-background, the $\epsilon$-deformed chiral correlator $\left\langle\operatorname{tr} \tilde{\varphi}^{J}(x)\right\rangle$ provides a simple class of supersymmetric observables of the gauge theory. Supersymmetric Ward identities show that these correlators are independent of the position $x$.

It is easy to see that the chiral correlators can be extracted from the deformed partition function. To this aim, we first introduce the $Q_{\xi}$-invariant volume form

$$
\alpha_{4}=d z_{1} d \bar{z}_{1} d z_{2} d \bar{z}_{2}-i\left(\epsilon_{1}\left|z_{1}\right|^{2} d z_{2} d \bar{z}_{2}+\epsilon_{2}\left|z_{2}\right|^{2} d z_{1} d \bar{z}_{1}\right)-\epsilon_{1} \epsilon_{2} z_{1} \bar{z}_{1} z_{2} \bar{z}_{2}
$$

and write the chiral operator in the equivariant form

$$
\int d^{4} x \operatorname{tr} \tilde{\varphi}^{J}=\int_{\mathbb{C}^{2}} \alpha_{4} \wedge \operatorname{tr} \mathcal{F}^{J}
$$

Using the localization formula (2.21) one finds for the normalized chiral correlator

$$
\left\langle\operatorname{tr} \tilde{\varphi}^{J}\right\rangle=\frac{\left\langle\int_{\mathbb{C}^{2}} \alpha_{4} \wedge \operatorname{tr} \mathcal{F}^{J}\right\rangle}{\left\langle\int_{\mathbb{C}^{2}} \alpha_{4}\right\rangle}=\frac{1}{Z_{\text {inst+tree }}} \sum_{Y} Z_{Y}(\vec{\tau}) \mathcal{O}_{J, Y}
$$

or equivalently from $(2.33)$

$$
\frac{1}{J !}\left\langle\operatorname{tr} \tilde{\varphi}^{J}\right\rangle=\frac{\mathrm{i} \epsilon_{1} \epsilon_{2}}{2 \pi} \partial_{\tau_{J}} \ln Z(\vec{\tau})
$$

Formula (2.39) generalizes the so called Matone relation that relates $\left\langle\operatorname{tr} \varphi^{2}\right\rangle$ to the $\tau$ derivative of the prepotential in the undeformed theory in flat space. On the other hand, it shows that one can view the deformed partition function as the generating functional of the general multi-trace chiral correlators

$$
\left\langle\operatorname{tr} \tilde{\varphi}^{J_{1}} \operatorname{tr} \tilde{\varphi}^{J_{2}} \ldots\right\rangle \text { undeformed }
$$

in the undeformed theory.

\section{The gauge theory on $S^{4}$}

The gauge partition function on $S^{4}$ is given by the integral [7]

$$
Z_{S^{4}}(\vec{\tau})=c \int_{\gamma} d^{N} a\left|Z_{\text {one-loop }}(a) Z_{\text {tree+inst }}(a, \vec{\tau})\right|^{2}
$$

with $d^{N} a=\prod_{u} d a_{u},{ }^{3}$ and $c$ a normalization constant. The integral runs along the imaginary axis. The partition functions $Z_{\text {one-loop }}$ and $Z_{\text {inst }}$ are given by (2.18) and (2.26) with vevs and masses taken in the domains

$$
a_{u}=\in \mathrm{i} \mathbb{R} \quad m, \epsilon_{\ell} \in \mathbb{R} \quad m_{u}=\frac{\epsilon}{2}+\mathrm{i} \mathbb{R} \quad \bar{m}_{u}=-\frac{\epsilon}{2}+\mathrm{i} \mathbb{R}
$$

\footnotetext{
${ }^{3}$ Notice that in our conventions the Vandermonde determinant is reabsorbed into the one-loop determinant $Z_{\text {one-loop }}$.
} 
These domains are chosen such that complex conjugate of the one-loop partition function (2.18) is given by the same formula with $\Gamma_{2}(x)$ replaced by $\Gamma_{2}(\epsilon-x)$. We adopt units where $\epsilon_{1} \epsilon_{2}=1$ and write $\epsilon_{1}=\epsilon_{2}^{-1}=b$. In these units the one-loop partition function becomes

$$
\begin{aligned}
\left|Z_{\text {one-loop }}^{\text {gauge }}\right|^{2} & =\prod_{u<v}^{N} \Upsilon\left(a_{u v}\right) \Upsilon\left(-a_{u v}\right) \\
\left|Z_{\text {one-loop }}^{\text {matter }}\right|^{2} & = \begin{cases}\prod_{u, v}^{N} \Upsilon\left(a_{u v}-m\right)^{-1} & \text { adj. } \\
\prod_{u, v=1}^{N} \Upsilon\left(a_{v}-\bar{m}_{u}\right)^{-1} \Upsilon\left(m_{v+N}-a_{u}\right)^{-1} & \text { fund. }\end{cases}
\end{aligned}
$$

with

$$
\Upsilon(x)=\frac{1}{\Gamma_{2}\left(x \mid b, \frac{1}{b}\right) \Gamma_{2}\left(b+\frac{1}{b}-x \mid b, \frac{1}{b}\right)}
$$

$\Upsilon$ is an entire function satisfying $\Upsilon(x)=\Upsilon(\epsilon-x)$. It has an infinite number of single zeros at $x=-m b-n / b$ and $x=(m+1) b+(n+1) b$ for $m, n \geq 0$ integers. Finally the normalization $c$ has been fixed for later convenience to be ${ }^{4}$

$$
c=q^{\frac{1}{2} m_{3}\left(m_{3}+\epsilon\right)+\frac{1}{2} m_{4}\left(m_{4}+\epsilon\right)}
$$

\subsection{Wilson loops}

A supersymmetric Wilson loop is defined by the line integral

$$
\mathcal{C}=\mathrm{i} \int_{0}^{L}\left(A_{m} \dot{x}^{m}+|\dot{x}| \varphi_{1}\right) d s
$$

with $\varphi_{1}=\frac{1}{2}\left(\varphi-\varphi^{\dagger}\right)$ and $A_{m}$ taken to be anti-hermitian matrices. We use complex coordinates $x^{m}=\left(z_{1}, z_{2}, \bar{z}_{1}, \bar{z}_{2}\right)$ and consider a circular Wilson loop defined by the path

$$
z_{\ell}(s)=r_{\ell} e^{\mathrm{i} \epsilon_{\ell} s}
$$

with

$$
L=\frac{2 \pi n_{1}}{\epsilon_{1}}=\frac{2 \pi n_{2}}{\epsilon_{2}}
$$

The condition (3.8) ensures that the path is closed and it can be satisfied if and only if the ratio $\epsilon_{1} / \epsilon_{2}$ is rational. Moreover, taking $r_{1,2}$ satisfying

$$
|\dot{x}|^{2}=\epsilon_{1}^{2}\left|r_{1}\right|^{2}+\epsilon_{2}^{2}\left|r_{2}\right|^{2}=1
$$

one finds

$$
\dot{x}^{m}=\left(\mathrm{i} \epsilon_{1} z_{1}, \mathrm{i} \epsilon_{2} z_{2},-\mathrm{i} \epsilon_{1} \bar{z}_{1},-\mathrm{i} \epsilon_{2} \bar{z}_{2}\right)=\delta_{\xi} x^{m}
$$

and the Wilson loop can be written in the suggestive form

$$
\mathcal{C}=\mathrm{i} \int_{0}^{L}\left(A_{m} \delta_{\xi} x^{m}+\varphi_{1}\right) d s=\frac{\mathrm{i}}{2} \int_{0}^{L} \tilde{\varphi}(s) d s+\text { h.c. }
$$

\footnotetext{
${ }^{4}$ This normalization is chosen in such a way that the partition function and its AGT dual correlator precisely match.
} 
The appearance of the combination $\tilde{\varphi}$ in the Wilson loop is not surprising, since this is the supersymmetric (equivariant) version of the vector field one form $A$. The evaluation of the Wilson loop expectation value can then be performed again with the help of localization by inserting in the partition function the operator tr $e^{\mathcal{C}}$. We notice that the 0 -form part $\mathcal{C}_{0}=\mathrm{i} \tilde{\varphi}_{0}=\mathrm{i} \bar{U} \delta_{\xi} U$ is anti-hermitian and that correlators of $\tilde{\varphi}(s)$ do not depend on $s$, so the Wilson loop operator on the instanton background $Y$ reduces to

$$
\left.e^{\mathcal{C}_{0}}\right|_{Y}=\left.\operatorname{tr} e^{\mathrm{i} L \tilde{\varphi}_{0}}\right|_{Y}=\sum_{u} e^{\frac{2 \pi \mathrm{i} n_{1}}{\epsilon_{1}} a_{u}}
$$

In deriving this relation we used (2.30) evaluated at $z=\frac{2 \pi \mathrm{i} n_{1}}{\epsilon_{1}}$. Remarkably, for this special value of $z$, the whole dependence of $\mathcal{C}$ on the instanton configuration $Y$ cancels upon exponentiation! The expectation value of the Wilson loop is then given by the simple formula

$$
\left\langle\operatorname{tr} e^{\mathcal{C}}\right\rangle_{S^{4}}=\frac{1}{Z} \int_{\gamma} d^{N} a \operatorname{tr} e^{\frac{2 \pi \mathrm{in} n_{1} a}{\epsilon_{1}}}\left|Z_{\text {one-loop }}(a) Z_{\text {tree+inst }}(a, \vec{\tau})\right|^{2}
$$

For the undeformed theory, formula (3.13) was proposed and motivated in [18] as the generalization of the more familiar result on the round sphere to the case of a squashed sphere with a Wilson loop oriented along the $z_{1}$-plane. This loop corresponds to the choice $r_{2}=0$ in (3.7). In this case there is no restriction on the sphere squashing parameter and the ratio $\epsilon_{1} / \epsilon_{2}$ is a continuos parameter. Our result provides a proof of that formula and a generalisation to the case of $\mathcal{N}=2$ theories with a classical prepotential of general type. We notice that $\tau_{J}$-corrections to the Wilson loop expectation value are computed by the insertions of chiral operators in the Wilson loop correlator, so that the expectation values of the Wilson loop in the deformed theory can be thought of as the generating function of the correlators

$$
\left\langle\operatorname{tr} e^{\mathcal{C}} \operatorname{tr} \tilde{\varphi}^{J_{1}} \tilde{\varphi}^{J_{2}} \ldots\right\rangle_{S^{4}, \text { undef. }}
$$

in the undeformed theory. For example, the first $\tau_{J}$-correction to the Wilson loop expectation value is computed by the correlator

$$
\frac{\partial}{\partial \tau_{J}}\left\langle\operatorname{tr} e^{\mathcal{C}}\right\rangle_{S^{4}}=\frac{2 \pi \mathrm{i}}{\epsilon_{1} \epsilon_{2} J !}\left\langle\operatorname{tr} e^{\mathcal{C}} \operatorname{tr} \tilde{\varphi}^{J}\right\rangle_{S^{4}, \text { undef. }}
$$

\subsubsection{Perturbative expansion}

The correlators (3.14), (3.15) can be computed order by order in perturbation theory in the weak coupling regime $\operatorname{Im} \tau \rightarrow \infty$. In this limit, the integral (3.13) is dominated by the region where $a$ is small. Expanding the integrand in (3.13) in powers of $a$, one can compute each term in the expansion in terms of a correlator in an effective matrix model. Using $\Upsilon(x) \sim x$ for $x$ small, and the fact that instanton corrections are suppressed in this limit one finds

$$
\left|Z_{\text {oneloop }}\right|^{2} \sim \Delta(a)=\prod_{u<v} a_{u v}^{2}+\ldots \quad Z_{\text {inst }}=1+\ldots
$$

Plugging (3.16) into (3.15) one finds, for the leading $\tau_{J}$-correction to the Wilson loop expectation value at weak coupling, the result

$$
\frac{\partial}{\partial \tau_{J}}\left\langle\operatorname{tr} e^{\mathcal{C}}\right\rangle_{S^{4}}=\frac{2 \pi \mathrm{i}}{\epsilon_{1} \epsilon_{2} J !} \int_{\gamma} d^{N} a \Delta(a) e^{\frac{2 \pi}{\epsilon_{1} \epsilon_{2}} \operatorname{Im} \tau \operatorname{tr} a^{2}} \operatorname{tr} a^{J} \operatorname{tr} a^{2}+\ldots
$$


Similarly, higher order corrections in perturbation theory are given by Gaussian matrix model integrals of type (3.17) involving higher order monomials in $a$. A direct test of these formulae by an explicit Feynman diagram computation would be very welcome.

\subsection{The $\mathcal{N}=4$ deformed theory}

In this section we consider the effect of turning on $\tau_{J}$-interactions on the simplest theory at our disposal: the $\mathcal{N}=4$ theory. $\tau_{J}$-deformations break $\mathcal{N}=4$ supersymmetry down to $\mathcal{N}=2$ by including self-interactions for one of the three chiral multiplets. The resulting theory is surprisingly simple and, as we will see, it exhibits a perfect cancellation of instanton contributions to the gauge partition function. This is in contrast with the case of $\mathcal{N}=2^{*}$ theory where the $\mathcal{N}=4$ symmetry is broken by giving mass to the adjoint hypermultiplet spoiling the balance between the instanton corrections coming from gauge and matter multiplets.

As in the undeformed case, the $\mathcal{N}=4$ deformed theory will be defined as a the limit of the $\mathcal{N}=2^{*}$ deformed theory where the mass of the adjoint hypermultiplet is sent to "zero" [7]. More precisely, the points where the $\mathcal{N}=4$ is restored will be identified with the zeros of the instanton partition function in the $m$-plane. It is easy to see that they are located at $m=-\epsilon_{1}$ or $m=-\epsilon_{2}$. Indeed, for any choice of $\tau_{J}$ and instanton number the instanton partition function (2.26) can be seen to be always proportional to $\left(m+\epsilon_{1}\right)\left(m+\epsilon_{2}\right)$ with the two factors coming from the contributions to $Z_{Y_{u} Y_{u}}(m)$ of the Young tableaux boxes $(i, j)=\left(k_{u j}, \tilde{k}_{u i}\right) \in Y_{u}$. We notice that these two points on the $m$-plane coincide in the case of the round sphere where $\epsilon_{1}=\epsilon_{2}=\frac{\epsilon}{2}$. and the symmetric point $m=-\frac{\epsilon}{2}$ is unique ( $m=0$ in the conventions of [7]). For concreteness here we take $m=-\epsilon_{1}$.

The one-loop partition function (2.17) also drastically simplifies at $m=-\epsilon_{1}$. Indeed using the double Gamma function identity

$$
\Gamma_{2}\left(x+\epsilon_{1}\right) \Gamma_{2}\left(x+\epsilon_{2}\right)=x \Gamma_{2}(x) \Gamma_{2}(x+\epsilon)
$$

one finds

$$
\left|Z_{\text {oneloop }}\right|^{2}=\Delta(a)=\prod_{u \neq v} a_{u v}
$$

with $\Delta(a)$ the Vandermonde determinant describing the $\mathrm{U}(N)$ measure. The gauge partition function reduces to the $\mathrm{U}(N)$ matrix model integrals

$$
Z=\int d^{N} a \Delta(a) e^{-N V(a, \vec{\tau})}
$$

with the integral over $a_{u}$ now running along the real line and the potential defined by

$$
V(a, \vec{\tau})=\frac{2 \pi \mathrm{i}}{\epsilon_{1} \epsilon_{2} N} \sum_{J=2}^{p} \frac{\tau_{J}}{J !} \operatorname{tr}(\mathrm{i} a)^{J}+\text { h.c. }
$$

On the other hand the expectation value of a circular Wilson loop is given by

$$
W=\frac{1}{Z} \int d^{N} a \Delta(a) \operatorname{tr} e^{\frac{2 \pi a}{\epsilon_{1}}} e^{-N V(a, \vec{\tau})}
$$


We notice that unlike in the $\mathcal{N}=4$ theory, in presence of $\tau_{J}$-interactions the matrix model underlying the theory is no-longer Gaussian but interacting. The integrals (3.20) and (3.22) count now diagrams involving not only propagators but also $J$-point vertices. Luckily enough, the underlying matrix models have been extensively studied in the literature. In the large $N$ limit integrals (3.20) and (3.22) have been explicitly evaluated by saddle point methods [5]. One defines the resolvent

$$
w(x)=\frac{1}{N}\left\langle\operatorname{tr} \frac{1}{x-a}\right\rangle=\frac{1}{N}\left\langle\sum_{u=1}^{N} \frac{1}{x-a_{u}}\right\rangle
$$

A simple algebra shows that $w(x)$ defined like this satisfies a quadratic equation with solution

$$
w(x)=\frac{1}{2}\left(V^{\prime}(x)-\sqrt{V^{\prime}(x)^{2}-4 f_{p-2}(x)}\right)
$$

where $f_{p-2}(x)$ a polynomial of order $p-2$ determined by the condition that $w(x) \approx \frac{1}{x}$ at large $|x|$. We notice that $w(x)$ has a discontinuity along the cuts defined by the zeroes of the square root, so we can write

$$
w(x \pm \mathrm{i} 0)=\int_{\mathcal{S}} \frac{\rho(y) d y}{x-y \pm \mathrm{i} 0}=\frac{1}{2} V^{\prime}(x) \pm \pi \mathrm{i} \rho(x)
$$

with $\mathcal{S}$ the union of the cuts and $\rho(x)$ the density

$$
\rho(x)=\frac{1}{N} \sum_{u} \delta\left(x-a_{u}\right)
$$

It is often enough to assume the presence of a single cut and look for $w(x)$ in the form

$$
w(x)=\frac{1}{2} V^{\prime}(x)-Q_{p-2}(x) \sqrt{\left(x-b_{1}\right)\left(x-b_{2}\right)}
$$

with $Q_{p-2}(x)$ a polynomial of order $p-2$. Indeed the number of unknown variables in $\left\{Q_{p-2}(x), b_{1}, b_{2}\right\}$ is $p+1$, matching the number of equations coming from requiring that $w(x) \approx \frac{1}{x}$ for large $|x|$ and therefore $w(x)$ is fully determined.

As an example, let us consider the quartic potential

$$
V(a)=\frac{1}{2 \lambda} a^{2}+g_{4} a^{4}
$$

with

$$
\lambda=\frac{N \epsilon_{1} \epsilon_{2}}{4 \pi\left(\operatorname{Im} \tau_{2}\right)}=\frac{g_{Y M}^{2} N \epsilon_{1} \epsilon_{2}}{16 \pi^{2}} \quad g_{4}=-\frac{4 \pi \operatorname{Im}\left(\tau_{4}\right)}{4 ! N \epsilon_{1} \epsilon_{2}}
$$

Since $V(a)=V(-a)$ one can take $b_{2}=-b_{1}=2 b$ and $Q(x)=c_{0}+c_{2} x^{2}$ in (3.27). Requiring $w(x) \approx \frac{1}{x}$ for large $|x|$ one finds

$$
w(x)=\frac{1}{2 \lambda}\left(x+4 x^{3} g_{4} \lambda-\left(1+4 \lambda g_{4}\left(x^{2}+2 b^{2}\right)\right) \sqrt{x^{2}-4 b^{2}}\right)
$$

with

$$
b^{2}=\frac{\sqrt{1+48 \lambda^{2} g_{4}}-1}{24 \lambda g_{4}}
$$




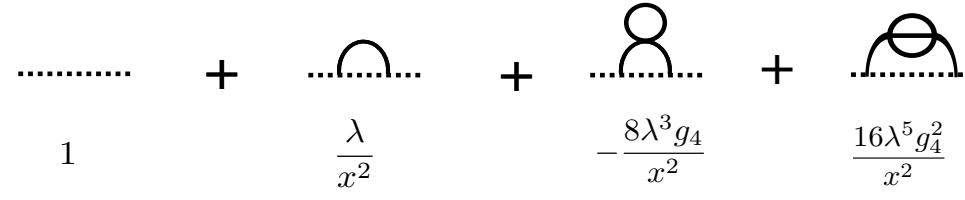

$$
\begin{aligned}
& \text { 8.... + } \\
& \frac{64 \lambda^{5} g_{4}^{2}}{x^{2}} \quad \frac{64 \lambda^{5} g_{4}^{2}}{x^{2}} \quad \frac{\lambda^{2}}{x^{4}} \quad \frac{\lambda^{2}}{x^{4}} \\
& \text { + ח... + } \\
& -\frac{4 \lambda^{4} g_{4}}{x^{4}} \quad-\frac{8 \lambda^{4} g_{4}}{x^{4}} \quad-\frac{8 \lambda^{4} g_{4}}{x^{4}} \quad-\frac{8 \lambda^{4} g_{4}}{x^{4}} \quad-\frac{8 \lambda^{4} g_{4}}{x^{4}}
\end{aligned}
$$

Figure 1. Diagrams contributing to the circular Wilson loops. The dashed line represents the $\tau$-line where Wilson loop operators $\mathcal{C}(\tau)$ are inserted.

Plugging (3.31) into (3.30) and expanding for large $x$ and small $\lambda$ one finds [5]

$$
x w(x)=\frac{1}{N} \sum_{n=0}^{\infty}\left\langle\frac{\operatorname{tr} a^{2 n}}{x^{2 n}}\right\rangle=1+\frac{\lambda-8 \lambda^{3} g_{4}+144 \lambda^{5} g_{4}^{2}}{x^{2}}+\frac{2 \lambda^{2}-36 \lambda^{4} g_{4}}{x^{4}}+\ldots
$$

Notice that only the even powers $\operatorname{tr} a^{2 n}$ have a non-trivial expectation value as expected for an even potential. Terms proportional to $x^{-2 n}\left(-g_{4}\right)^{k} \lambda^{p}$ count the number of diagrams with $2 n$ external lines, $p$ propagators and $k$ four-point vertices. In figure 1 we show the relevant diagrams and contributions leading to (3.32). The entire sum can be written in the analytic form [5]

$$
x w(x)=1+\sum_{n=1}^{\infty} \sum_{k=0}^{\infty} \frac{\left(-12 g_{4} \lambda^{2}\right)^{k} \lambda^{n}(2 n) !(2 k+n-1) !}{x^{2 n} n !(n-1) ! k !(k+n+1) !}
$$

For the Wilson loop one then finds

$$
\begin{aligned}
W & =\frac{1}{N} \sum_{n=0}^{\infty}\left(\frac{2 \pi n_{1}}{\epsilon_{1}}\right)^{2 n}\left\langle\operatorname{tr} \frac{a^{2 n}}{(2 n) !}\right\rangle \\
& =1+\sum_{n=1}^{\infty} \sum_{k=0}^{\infty} \frac{\left(-12 g_{4} \lambda^{2}\right)^{k}\left(\frac{4 \pi^{2} n_{1}^{2} \lambda}{\epsilon_{1}^{2}}\right)^{n}(2 k+n-1) !}{n !(n-1) ! k !(k+n+1) !} \\
& =\frac{2}{\sqrt{\lambda_{\text {eff }}}}\left[I_{1}\left(\sqrt{\lambda_{\text {eff }}}\right)+12 g_{4} b^{4} I_{3}\left(\sqrt{\lambda_{\text {eff }}}\right)\right]
\end{aligned}
$$

with

$$
\lambda_{\mathrm{eff}}=\frac{16 \pi^{2} n_{1}^{2} b^{2}}{\epsilon_{1}^{2}}=g_{Y M}^{2} N n_{1} n_{2} \frac{b^{2}}{\lambda}
$$


Formula (3.34) gives the large $N$ limit of the Wilson loop expectation value in the $\tau_{4^{-}}$ deformed $\mathcal{N}=4$ theory and it is exact in $\lambda_{\text {eff }}$. When $g_{4} \approx 0$ and $n_{1} n_{2}=1$ one finds the familiar $\mathcal{N}=4$ formula $[1,4,6]$

$$
W \approx \frac{2 I_{1}\left(\sqrt{\lambda_{\mathrm{YM}}}\right)}{\sqrt{\lambda_{\mathrm{YM}}}} \quad \text { with } \quad \lambda_{\mathrm{YM}}=g_{\mathrm{YM}}^{2} N
$$

On the other hand in the limit of large $\lambda_{\text {eff }}$ with $g_{4} \lambda^{2}$ kept finite one finds the same exponential growth of the $\mathcal{N}=4$ case but with $\lambda_{Y M}$ replaced by $\lambda_{\text {eff }}$. This result provides us with a remarkable simple prediction for the AdS dual of the deformed theory. Indeed according to holography, in this limit the minimal area of a string world sheet on AdS ending on the loop is given by $\ln W$ so one expects

$$
S=\ln W \approx \sqrt{\lambda_{\mathrm{eff}}}+\ldots
$$

On the gravity side, a general class of solutions preserving half of the supersymmetries of $A d S_{5} \times S^{5}$ were constructed in [34]. These solutions are specified by a single function $w(x)$ with branch cuts in the x-plane. It is natural to identify the gravity dual of the deformed $\mathcal{N}=4$ theory with the one-cut solution specified by the matrix model resolvent $w(x)$. The minimal string world sheet area in the $w$-geometry can be computed along the lines of [34] providing a precise test of the duality.

\subsection{Two point correlators $\left\langle\operatorname{tr} e^{\mathcal{C}} \operatorname{tr} \varphi^{J}\right\rangle$}

The expectation value of the Wilson loop in the deformed theory can be viewed as the generating function of correlators in the undeformed gauge theory involving the insertions of chiral primary operators in the Wilson loop. These correlators compute the expansion coefficients of chiral primary operators in the OPE of the circular Wilson at distances much larger than the size of the loop. In [20], correlators $\left\langle\operatorname{tr} e^{\mathcal{C}} \operatorname{tr} \varphi^{J}\right\rangle$ computing the leading coefficients in the expansion were evaluated for the $\mathcal{N}=4$ theory on $\mathbb{R}^{4}$. In this section we show how these results for $J=2,4$ can be extracted from our localization formulae.

The crucial observation in [20] is that the two point function $\left\langle A_{M} \dot{x}^{M}(x) A_{N} \dot{x}^{N}(y)\right\rangle$ with $x^{M}(s)$ the ten-dimensional path along the circular loop does not depend on the insertion point positions. Moreover, the contributions to the correlators $\left\langle\operatorname{tr} e^{\mathcal{C}} \operatorname{tr} \varphi^{J}\right\rangle$ coming from Feynman diagrams involving internal vertex insertions are argued to cancel to all orders in perturbation theory. The correlator is then effectively computed by a Gaussian matrix model with the insertion of a J-point vertex counting the number or rainbow diagrams ending on the loop with the extra J-point insertion. The same matrix model computes the leading $\tau_{J}$-correction of the deformed $\mathcal{N}=4$ gauge theory on $S^{4}$ in the limit where all $\tau_{J}$ 's are small. The results for the correlators for $J=2,4$ can then be extracted from the expansion of the exact formula (3.34) in the limit where $\tau_{2} \approx \tau$ and $\tau_{4} \approx 0$ i.e. the undeformed theory.

In doing so, we should remember that the chiral primary operators involved in the correlator are traceless, so the diagrams in figure 1 and figure 2 with loops starting and ending on the same four-point vertex should be discarded. Taking $\epsilon_{1}=\epsilon_{2}=4 \pi$ (3.29) reads

$$
\lambda=\lambda_{\mathrm{YM}}
$$



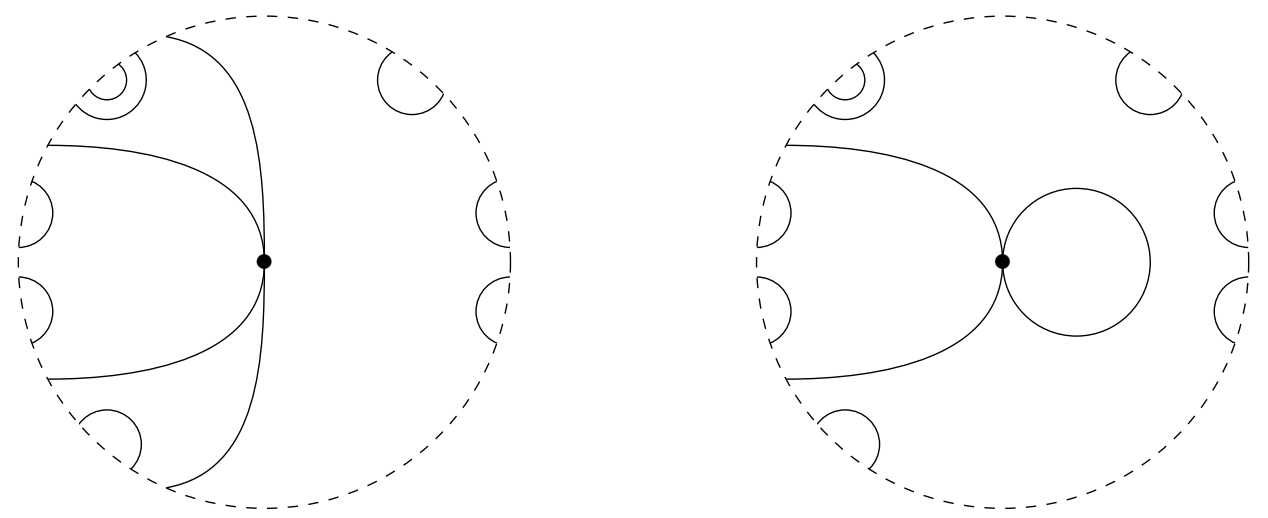

Figure 2. The left figure displays a typical diagram contributing to the correlator $\left\langle W \operatorname{tr} \varphi^{4}\right\rangle$. The dashed line stays for the Wilson loop. Solid lines denote gluon/scalar propagators. The correlator counts graphs involving a single quartic vertex interaction with all four legs ending on the Wilson loop. The counting discards diagrams of the type in the right figure where a pair of legs starting from the quartic vertex contract among themselves.

The correlators $\left\langle W \operatorname{tr} \varphi^{J}\right\rangle$ can be extracted from the small $g_{4}$-expansion of (3.34)

$$
W=W_{0}-g_{4} W_{1}+\ldots
$$

with

$$
\begin{aligned}
& W_{0}=\frac{2 I_{1}\left(\sqrt{\lambda_{\mathrm{YM}}}\right)}{\sqrt{\lambda_{\mathrm{YM}}}}=1+\frac{\lambda_{\mathrm{YM}}}{42 !}+\frac{2 \lambda_{\mathrm{YM}}^{2}}{4^{2} 4 !}+\frac{5 \lambda_{\mathrm{YM}}^{3}}{4^{3} 6 !}+\ldots \\
& W_{1}=12 \lambda_{\mathrm{YM}}^{2} I_{4}\left(\sqrt{\lambda_{\mathrm{YM}}}\right)+48 \lambda_{\mathrm{YM}}^{\frac{3}{2}} I_{3}\left(\sqrt{\lambda_{\mathrm{YM}}}\right)=\frac{8 \lambda_{\mathrm{YM}}^{3}}{42 !}+\frac{36 \lambda_{\mathrm{YM}}^{4}}{4^{2} 4 !}+\frac{144 \lambda_{\mathrm{YM}}^{5}}{4^{3} 6 !}+\ldots
\end{aligned}
$$

The integer coefficients in the numerators of the right hand side expansions (3.40) count the number of diagrams with zero and one $g_{4}$-vertices in figure 1 . The insertion of $\operatorname{tr} \varphi^{2}$ inside the Wilson loop correlator can be obtained from $W_{0}$ after taking a derivative with respect to $\lambda$. Indeed using (3.22) and (3.28) one finds

$$
\left\langle\operatorname{tr} e^{\mathcal{C}} \operatorname{tr} \varphi^{2}\right\rangle=2 \lambda_{\mathrm{YM}}^{2} \frac{\partial}{\partial \lambda_{\mathrm{YM}}} W_{0}=2 \lambda_{\mathrm{YM}} I_{2}\left(\sqrt{\lambda_{\mathrm{YM}}}\right)
$$

and it agrees with the result in [20]. The result for $\left\langle\operatorname{tr} e^{\mathcal{C}} \operatorname{tr} \varphi^{4}\right\rangle$ follows from $W_{1}$ after subtracting the contributions of the diagrams involving four-point vertices with self contracted legs. These diagrams are counted by

$$
8 \lambda_{\mathrm{YM}}^{3} \frac{\partial}{\partial \lambda_{\mathrm{YM}}} W_{0}=8 \lambda_{\mathrm{YM}} I_{2}\left(\sqrt{\lambda_{\mathrm{YM}}}\right)=\frac{8 \lambda_{\mathrm{YM}}^{3}}{42 !}+\frac{32 \lambda_{\mathrm{YM}}^{4}}{4^{2} 4 !}+\frac{128 \lambda_{\mathrm{YM}}^{5}}{4^{3} 6 !}+\ldots
$$

Subtracting this contribution from $W_{1}$ one finds

$$
\left\langle\operatorname{tr} e^{\mathcal{C}} \operatorname{tr} \varphi^{4}\right\rangle=\frac{\lambda_{\mathrm{YM}}^{2}}{4} I_{4}\left(\sqrt{\lambda_{\mathrm{YM}}}\right)=\frac{4 \lambda_{\mathrm{YM}}^{4}}{4^{2} 4 !}+\frac{16 \lambda_{\mathrm{YM}}^{5}}{4^{3} 6 !}+\ldots
$$

in agreement with [20]. Our derivation, based on localization, can be considered as a proof of the formulae derived in [20] under the assumptions that correlators $\left\langle\operatorname{tr} e^{\mathcal{C}} \operatorname{tr} \varphi^{J}\right\rangle$ are not 
corrected by graphs involving internal vertex interactions. The results provide exact (to all orders in perturbation theory at large N) formulae for correlators involving Wilson loops and chiral primary operators generalizing the Wilson loop results obtained in [7] using localization and in $[1,4,6]$ from perturbation theory.

\section{AGT duality: chiral correlators vs integrals of motion}

\subsection{The CFT side}

The AGT correspondence [17] relates $\mathcal{N}=2$ supersymmetric gauge theories in four dimensions to two dimensional CFTs. According to this correspondence the instanton partition function for the $\mathrm{SU}(2)$ gauge theory with four fundamentals is related to four-point correlators in Liouville theory. In this section we study the CFT side of the duality. In the next section we will study the gauge side of it and show that the chiral correlators $\left\langle\operatorname{tr} \tilde{\varphi}^{n}\right\rangle$ in the gauge theory are reproduced by the same four-point correlators in Liouville theory with the insertion of the integrals of motion $I_{n}$ introduced in [35].

\subsubsection{The conformal field theory}

Here we follow [35]. We refer the reader to this paper for details and further references. The symmetry algebra of Liouville theory is the tensor product of a Virasoro and a Heisenberg algebra with commutation relations

$$
\begin{aligned}
{\left[L_{m}, L_{n}\right] } & =(m-n) L_{m+n}+\frac{c}{12}\left(m^{3}-m\right) \delta_{m+n, 0} \\
{\left[a_{m}, a_{n}\right] } & =\frac{m}{2} \delta_{m+n} \quad\left[L_{m}, a_{n}\right]=0
\end{aligned}
$$

The central charge $\mathrm{c}$ is parametrized by

$$
c=1+6 Q^{2} \quad \text { where } \quad Q=b+\frac{1}{b}
$$

The primary fields $V_{\alpha}$ are defined as

$$
V_{\alpha}(z)=\mathcal{V}_{\alpha}^{\mathrm{vir}}(z) \mathcal{V}_{\alpha}^{\text {heis }}(z)
$$

with $\mathcal{V}_{\alpha}^{\text {vir }}$ a primary field of the Virasoro algebra with dimension $\Delta(\alpha)=\alpha(Q-\alpha)$ and

$$
\mathcal{V}_{\alpha}^{\text {heis }}(z)=e^{2 i(\alpha-Q) \sum_{n<0} \frac{a_{n}}{n} z^{-n}} e^{2 i \alpha \sum_{n>0} \frac{a_{n}}{n} z^{-n}}
$$

The commutation relations of the field $V_{\alpha}$ and the generators $L_{m}, a_{n}$ are

$$
\begin{aligned}
{\left[L_{m}, V_{\alpha}(z)\right] } & =\mathcal{V}_{\alpha}^{\text {heis }}(z)\left(z^{m+1} \partial_{z}+(m+1) \Delta(\alpha) z^{m}\right) \mathcal{V}_{\alpha}^{\mathrm{vir}}(z) \\
{\left[a_{n}, V_{\alpha}(z)\right] } & =\left\{\begin{array}{cll}
\mathrm{i}(Q-\alpha) z^{n} V_{\alpha}(z) & \text { for } & n>0 \\
-\mathrm{i} \alpha z^{n} V_{\alpha}(z) & \text { for } & n<0
\end{array}\right.
\end{aligned}
$$

The Fock space is obtained by acting with $L_{n}, a_{n}$ with $n<0$ on a vacuum $|0\rangle$ defined by

$$
L_{m}|0\rangle=a_{n}|0\rangle=0 \quad \text { for } \quad m \geq-1, \quad n>0
$$


Primary states $|\alpha\rangle$ and $\langle\alpha|$ are obtained by acting on the vacuum with the primary fields at zero and infinity respectively

$$
|\alpha\rangle=V_{\alpha}(0)|0\rangle \quad\langle\alpha|=\lim _{z \rightarrow \infty} z^{2 \Delta(\alpha)}\langle 0| V_{\alpha}(z)
$$

Any correlator of the composite fields $V_{\alpha}$ factorizes into the product of a Heisenberg and a Virasoro part. The Heisenberg part, which are just free bosons, is easy to compute and it reads

$$
\left\langle\mathcal{V}_{\alpha_{1}}^{\mathrm{heis}}\left(z_{1}\right) \mathcal{V}_{\alpha_{2}}^{\mathrm{heis}}\left(z_{2}\right) \mathcal{V}_{\alpha_{3}}^{\mathrm{heis}}\left(z_{3}\right) \mathcal{V}_{\alpha_{4}}^{\mathrm{heis}}\left(z_{4}\right)\right\rangle=\left(1-\frac{z_{3}}{z_{2}}\right)^{2 \alpha_{2}\left(Q-\alpha_{3}\right)}
$$

Consider the remaining Virasoro part of the four-point correlator

$$
\mathcal{G}_{\text {vir }}\left(\alpha_{i}, \alpha \mid z_{i}\right)=z_{1}^{2 \Delta\left(\alpha_{1}\right)}\left\langle\left\langle\mathcal{V}_{\alpha_{1}}^{\mathrm{vir}}\left(z_{1}\right) \mathcal{V}_{\alpha_{2}}^{\mathrm{vir}}\left(z_{2}\right) \mathcal{V}_{\alpha_{3}}^{\mathrm{vir}}\left(z_{3}\right) \mathcal{V}_{\alpha_{4}}^{\mathrm{vir}}\left(z_{4}\right)\right\rangle\right\rangle_{\alpha}
$$

where by $\langle\langle\rangle\rangle_{\alpha}$ we denote the four-point conformal block involving the exchange of a state of conformal dimension

$$
\Delta=\Delta(\alpha)
$$

and the factor $z_{1}^{2 \Delta\left(\alpha_{1}\right)}$ is included to guarantee a finite limit at $z_{1} \rightarrow \infty$. The fact that the 4-point correlator depends non-trivially only on the cross ratio follows from conformal invariance, so without loss of generality we can fix three points, let us say $z_{1}=\infty, z_{2}=1$ and $z_{4}=0$ and denote the resulting function of a single variable $z \equiv z_{3}$ as $\mathcal{G}_{\text {vir }}\left(\alpha_{i}, \alpha \mid z\right)$ or simply as $\mathcal{G}_{\text {vir }}$ if it is clear from the context, what are the argument and the parameters. Derivatives $\partial_{z_{i}} \mathcal{G}_{\text {vir }}$ of the correlator can be also written in terms of derivative with respect to $z$. For the choice above one finds [32]

$$
\begin{array}{ll}
\partial_{z_{1}} \mathcal{G}_{\text {vir }}=0 & \partial_{z_{2}} \mathcal{G}_{\text {vir }}=\left(-z \partial_{z}+2 \Delta_{1}-\delta\right) \mathcal{G}_{\text {vir }} \\
\partial_{z_{3}} \mathcal{G}_{\text {vir }}=\partial_{z} \mathcal{G}_{\text {vir }} & \partial_{z_{4}} \mathcal{G}_{\text {vir }}=\left((z-1) \partial_{z}+\delta-2 \Delta_{1}\right) \mathcal{G}_{\text {vir }}
\end{array}
$$

with $\delta=\sum_{i=1}^{4} \Delta_{i}$ and $\Delta_{i}=\Delta\left(\alpha_{i}\right)$. Including also the contribution of the Heisenberg sector one finds the conformal block

$$
\mathcal{G}\left(\alpha_{i}, \alpha \mid z\right) \equiv\left\langle\left\langle\alpha_{1}\left|V_{\alpha_{2}}(1) V_{\alpha_{3}}(z)\right| \alpha_{4}\right\rangle\right\rangle_{\alpha}=(1-z)^{2 \alpha_{2}\left(Q-\alpha_{3}\right)} \mathcal{G}_{\text {vir }}\left(\alpha_{i}, \alpha \mid z\right)
$$

The "physical" correlator can be written as the integral of the modulus square of the conformal block (4.12)

$$
G\left(\alpha_{i} \mid z\right)=\int \frac{d \alpha}{2 \pi} C_{\alpha_{1} \alpha_{2} \alpha} C_{\alpha \alpha_{3} \alpha_{4}}\left|\mathcal{G}\left(\alpha_{i}, \alpha \mid z\right)\right|^{2}
$$

where $C_{\alpha_{1} \alpha_{2} \alpha}$ are the Liouville structure constants [36, 37]. 


\subsubsection{Integrals of motion}

Being an integrable system, the Liouville theory admits the existence of an infinite set of mutually commuting operators or integrals of motion. Explicitly the first three such integrals are [35]

$$
\begin{aligned}
I_{2}= & L_{0}-\frac{c}{24}+2 \sum_{k=1}^{\infty} a_{-k} a_{k} \\
I_{3}= & \sum_{k=-\infty, k \neq 0}^{\infty} a_{-k} L_{k}+2 \mathrm{i} Q \sum_{k=1}^{\infty} k a_{-k} a_{k}+\frac{1}{3} \sum_{i+j+k=0} a_{i} a_{j} a_{k} \\
I_{4}= & 2 \sum_{k=1}^{\infty} L_{-k} L_{k}+L_{0}^{2}-\frac{c+2}{12} L_{0}+6 \sum_{k=-\infty, k \neq 0}^{\infty} \sum_{i+j=k} L_{-k} a_{i} a_{j}+12\left(L_{0}-\frac{c}{24}\right) \sum_{k=1}^{\infty} a_{-k} a_{k} \\
& +6 \mathrm{i} Q \sum_{k=-\infty, k \neq 0}^{\infty}|k| a_{-k} L_{k}+2\left(1-5 Q^{2}\right) \sum_{k=1}^{\infty} k^{2} a_{-k} a_{k}+6 \mathrm{i} Q \sum_{i+j+k=0}|k| a_{i} a_{j} a_{k} \\
& +\sum_{i+j+k+l=0}: a_{i} a_{j} a_{k} a_{l}:
\end{aligned}
$$

We can insert these operators inside the four-point correlators and the corresponding conformal blocks. We define

$$
\mathcal{G}_{n}\left(\alpha_{i}, \alpha \mid z\right)=\left\langle\left\langle\alpha_{1}\left|V_{\alpha_{2}}(1) I_{n} V_{\alpha_{3}}(z)\right| \alpha_{4}\right\rangle\right\rangle_{\alpha}
$$

To compute $\mathcal{G}_{n}$, we can use the commutation relations (4.5) to bring creator and annihilator operators to the left and right sides of the correlation respectively. For instance ${ }^{5}$

$$
\begin{aligned}
\mathcal{G}_{2} & =\left\langle\left\langle\alpha_{1}\left|V_{\alpha_{2}}(1)\left[L_{0}, V_{\alpha_{3}}(z)\right]\right| \alpha_{4}\right\rangle\right\rangle_{\alpha}+2 \sum_{k=1}^{\infty}\left\langle\left\langle\alpha_{1}\left|\left[V_{\alpha_{2}}(1), a_{-k}\right]\left[a_{k}, V_{\alpha_{3}}(z)\right]\right| \alpha_{4}\right\rangle\right\rangle_{\alpha}+\left(\Delta_{4}-\frac{c}{24}\right) \mathcal{G} \\
& =\left(z \partial_{z}+\frac{2 \alpha_{2}\left(Q-\alpha_{3}\right) z}{1-z}+\Delta_{3}+\Delta_{4}-\frac{c}{24}-\frac{2 \alpha_{2}\left(Q-\alpha_{3}\right) z}{1-z}\right) \mathcal{G} \\
& =\left(z \partial_{z}+\Delta_{3}+\Delta_{4}-\frac{c}{24}\right) \mathcal{G}
\end{aligned}
$$

Similarly for $\mathcal{G}_{3}$ one finds

$$
\begin{aligned}
\mathcal{G}_{3} & =\mathrm{i} \sum_{k=1}^{\infty} z^{k}\left[z\left(Q+\alpha_{2}-\alpha_{3}\right)\left(\partial_{z}+\frac{2 \alpha_{2}\left(Q-\alpha_{3}\right)}{1-z}\right)+(k+1) \alpha_{2} \Delta_{3}+(k-1)\left(Q-\alpha_{3}\right) \Delta_{2}\right] \mathcal{G} \\
& \mathrm{i}\left[-2 Q \alpha_{2}\left(Q-\alpha_{3}\right) \sum_{k=1}^{\infty} z^{k}+\alpha_{2}\left(Q-\alpha_{3}\right)\left(Q+\alpha_{2}-\alpha_{3}\right) \sum_{i, j=1}^{\infty} z^{i+j}\right] \mathcal{G} \\
& \left.=\frac{\mathrm{i} z}{1-z}\left[\left(Q+\alpha_{2}-\alpha_{3}\right) z \partial_{z}+\left(Q-\alpha_{3}\right)\left(\Delta_{2}+\Delta_{3}+\Delta_{4}-\Delta_{1}\right)-2 \alpha_{2}\left(Q-\alpha_{3}\right)^{2}\right)\right] \mathcal{G}
\end{aligned}
$$

\footnotetext{
${ }^{5}$ One should be careful to take into account that the commutators $\left[L_{n}, V_{\alpha}\right]$ produce derivatives only of the Virasoro part of the composite field $V_{\alpha}$.
} 
Proceeding in this way one can write $\mathcal{G}_{n}$ in terms of $\mathcal{G}$ and their $z$-derivatives, or as differential operators acting on $\mathcal{G}$. We write

$$
\mathcal{G}_{n}\left(\alpha_{i}, \alpha \mid z\right)=\mathcal{L}_{n} \mathcal{G}\left(\alpha_{i}, \alpha \mid z\right)
$$

with $\mathcal{L}_{n}$ a differential operator on the variable $z$. For the first few terms one finds

$$
\begin{aligned}
& \mathcal{L}_{2}=z \partial_{z}+\Delta_{3}+\Delta_{4}-\frac{c}{24} \\
& \mathcal{L}_{3}=\frac{i z}{1-z}\left[\left(Q+\alpha_{2}-\alpha_{3}\right) z \partial_{z}+\left(Q-\alpha_{3}\right)\left(\Delta_{2}+\Delta_{3}+\Delta_{4}-\Delta_{1}\right)-2 \alpha_{2}\left(Q-\alpha_{3}\right)^{2}\right]
\end{aligned}
$$

We have also computed an analogous formula for $\mathcal{L}_{4}$, but it is too lengthy to be reproduced here. Instead, later we will present its gauge theory counterpart, which is less cumbersome (see eq. (4.26)).

\subsection{The gauge/CFT dictionary}

The AGT correspondence relates the four-point conformal block of the Liouville theory to the partition function of the $\mathcal{N}=2$ supersymmetric $\mathrm{SU}(2)$ gauge theory with four fundamentals. The gauge coupling parameter $q=e^{2 \pi \mathrm{i} \tau}$ is identified with the harmonic ratio $z$ parametrizing the positions of vertex insertions. The gauge theory masses $m_{u}, \bar{m}_{2+u}$ are related to the conformal dimensions of the vertex insertions in the CFT. To achieve a full symmetry with respect to the exchange of the four masses we make the replacements $m_{3} \rightarrow \bar{m}_{3}+\epsilon, m_{4} \rightarrow \bar{m}_{4}+\epsilon$. The vacuum expectation value $a$ for the scalar field at infinity parametrizes the dimension of the exchanged state. The squeezing parameter $\epsilon_{1} / \epsilon_{2}$ characterizing the $\Omega$ gravitational background parametrizes the central charge of the CFT. The full dictionary is given by [17]

$$
\begin{aligned}
\alpha_{1} & =\frac{\epsilon}{2}+\frac{1}{2}\left(\bar{m}_{1}-\bar{m}_{2}\right) & \alpha_{2} & =-\frac{1}{2}\left(\bar{m}_{1}+\bar{m}_{2}\right) \\
\alpha_{3} & =\epsilon+\frac{1}{2}\left(\bar{m}_{3}+\bar{m}_{4}\right) & \alpha_{4} & =\frac{\epsilon}{2}+\frac{1}{2}\left(\bar{m}_{3}-\bar{m}_{4}\right) \\
\alpha & =\frac{\epsilon}{2}+a \quad \epsilon=\epsilon_{1}+\epsilon_{2}=Q & \epsilon_{1} & =b \quad \epsilon_{2}=b^{-1} \quad z=q
\end{aligned}
$$

The instanton partition function of the gauge theory on $\mathbb{R}^{4}$ is related to the conformal block $\mathcal{G}\left(\alpha_{i}, \alpha \mid q\right)$ via

$$
Z_{\text {tree+inst }}^{\mathrm{U}(2)}\left(a, m_{i}, q\right)=q^{-a^{2}} Z_{\text {inst }}^{\mathrm{U}(2)}\left(a, m_{i}, q\right)=q^{-\frac{Q^{2}}{4}+\Delta_{3}+\Delta_{4}} \mathcal{G}\left(\alpha, \alpha_{i}, q\right)
$$

with $Z_{\text {inst }} \sim 1$ and $\mathcal{G} \sim q^{\Delta-\Delta_{3}-\Delta_{4}}$ for small $q$. On the other hand, the Virasoro conformal block is related to the $\mathrm{SU}(2)$ partition function via

$$
\mathcal{G}_{\text {vir }}\left(\alpha, \alpha_{i}, q\right)=q^{\Delta-\Delta_{3}-\Delta_{4}} Z_{\text {inst }}^{\mathrm{SU}(2)}\left(a, m_{i} q\right)=q^{\Delta-\Delta_{3}-\Delta_{4}}(1-q)^{-2 \alpha_{2}\left(Q-\alpha_{3}\right)} Z_{\text {inst }}^{\mathrm{U}(2)}\left(a, m_{i} q\right)
$$

with the extra factor canceling the U(1) contribution (4.8) arising from the Heisenberg CFT field. The full four-point correlator (4.13) is then identified with the gauge partition function on the sphere via

$$
G\left(\alpha_{i}, q\right)=Z_{S^{4}}^{\mathrm{U}(2)}\left(m_{i}, q\right)
$$




\subsection{The gauge theory side}

It is known [21-23] that the integrals of motion (4.14) can be put in relation to the chiral correlators $\left\langle\operatorname{tr} \tilde{\varphi}^{J}\right\rangle$. In this section we translate (4.19) in terms of the gauge theory variables to find that the chiral correlators in the undeformed $\mathrm{U}(2)$ gauge theory can be expressed in terms of q-derivatives of the partition function $Z$. This leads to chiral ring type relations valid at all-instanton orders for a finite $\Omega$-background. The results will be checked against a microscopic instanton computation and in the so called Nekrasov-Shatashvili limit, where one of the two parameters of the $\Omega$-background goes to zero, with the analog of the SeibergWitten curve obtained in $[25,26]$.

\subsubsection{Chiral relations: $\epsilon_{1}, \epsilon_{2}$ finite}

The results (4.18) and (4.19) can be translated into chiral correlators using the identification

$$
\begin{aligned}
& \left\langle\operatorname{tr} \tilde{\varphi}^{2}\right\rangle=-2 \frac{\mathcal{G}_{2}(q)}{\mathcal{G}}-\frac{1}{12} \\
& \left\langle\operatorname{tr} \tilde{\varphi}^{3}\right\rangle=6 \mathrm{i} \frac{\mathcal{G}_{3}(q)}{\mathcal{G}} \\
& \left\langle\operatorname{tr} \tilde{\varphi}^{4}\right\rangle=2 h^{4} \frac{\mathcal{G}_{4}(q)}{\mathcal{G}}-\frac{h^{2}}{4}\left\langle\operatorname{tr} \tilde{\varphi}^{2}\right\rangle+\frac{\epsilon^{2}\left(h^{2}+\epsilon^{2}\right)}{8}
\end{aligned}
$$

where

$$
\epsilon=\epsilon_{1}+\epsilon_{2} \quad h^{2}=\epsilon_{1} \epsilon_{2}
$$

Using the AGT dictionary (4.20), (4.21) leads to

$$
\begin{aligned}
\left\langle\operatorname{tr} \tilde{\varphi}^{2}\right\rangle= & -2 h^{2} \frac{q \partial_{q} Z}{Z} \\
\left\langle\operatorname{tr} \tilde{\varphi}^{3}\right\rangle= & \frac{3 q}{1-q}\left(-h^{2} M_{1} \frac{q \partial_{q} Z}{Z}+M_{3}\right) \\
\left\langle\operatorname{tr} \tilde{\varphi}^{4}\right\rangle= & \frac{2 q}{(1-q)^{2}}\left(2 \epsilon M_{3}+2 M_{4}+2 q\left(M_{1} M_{3}-M_{4}\right)+h^{4} q\left(1-q^{2}\right) \frac{\partial_{q}^{2} Z}{Z}\right. \\
& \left.+h^{2}\left[h^{2}-2 q\left(\epsilon M_{1}+M_{2}\right)+q^{2}\left(-h^{2}+2 M_{2}-2 M_{1}^{2}\right)\right] \frac{\partial_{q} Z}{Z}\right)
\end{aligned}
$$

with $Z=Z_{\text {one-loop }}^{\mathrm{U}(2)} Z_{\text {inst+tree }}^{\mathrm{U}(2)}$ and

$$
M_{1}=-\sum_{i=1}^{4} \bar{m}_{i} \quad M_{2}=\sum_{i<j}^{4} \bar{m}_{i} \bar{m}_{j} \quad M_{3}=-\sum_{i<j<k}^{4} \bar{m}_{i} \bar{m}_{j} \bar{m}_{k} \quad M_{4}=\bar{m}_{1} \bar{m}_{2} \bar{m}_{3} \bar{m}_{4}
$$

We notice that the last two equations of (4.26) can be rewritten in the form

$$
\begin{aligned}
\left\langle\operatorname{tr} \tilde{\varphi}^{3}\right\rangle= & \frac{3 q}{1-q}\left(\frac{1}{2}\left\langle\operatorname{tr} \tilde{\varphi}^{2}\right\rangle M_{1}+M_{3}\right) \\
\left\langle\operatorname{tr} \tilde{\varphi}^{4}\right\rangle= & \frac{(1+q)}{2(1-q)}\left\langle\operatorname{tr} \tilde{\varphi}^{2}\right\rangle^{2}+\frac{2 q}{(1-q)^{2}}\left(M_{2}(1-q)+q M_{1}^{2}+\epsilon M_{1}\right)\left\langle\operatorname{tr} \tilde{\varphi}^{2}\right\rangle \\
& -h^{2} \frac{1+q}{1-q} q \partial_{q}\left\langle\operatorname{tr} \tilde{\varphi}^{2}\right\rangle+\frac{4 q}{(1-q)^{2}}\left(M_{4}(1-q)+q M_{1} M_{3}+\epsilon M_{3}\right)
\end{aligned}
$$


which shows that in a finite $\Omega$-background, chiral correlators can be written in terms of both $\left\langle\operatorname{tr} \tilde{\varphi}^{2}\right\rangle$ and its derivatives. The chiral ring equations (4.28) generalize those found in [24] to the case of finite $\epsilon_{1}, \epsilon_{2}$. The relations (4.26) can be checked against a microscopic instanton computation. Using (2.26)-(2.39) one finds

$$
\begin{aligned}
\left\langle\operatorname{tr} \tilde{\varphi}^{2}\right\rangle= & 2 a^{2}+\frac{2 q}{4 a^{2}-\epsilon^{2}}\left(2 a^{4}+a^{2}\left(-\epsilon M_{1}+2 M_{2}\right)-\epsilon M_{3}+2 M_{4}\right)+\ldots \\
\left\langle\operatorname{tr} \tilde{\varphi}^{3}\right\rangle= & q\left(3 a^{2} M_{1}+3 M_{3}\right)+\ldots \\
\left\langle\operatorname{tr} \tilde{\varphi}^{4}\right\rangle= & 2 a^{4}+\frac{2 q}{4 a^{2}-\epsilon^{2}}\left[2 a^{2}\left(6 a^{2}-h^{2}-\epsilon^{2}\right)\left(a^{2}+M_{2}\right)+\epsilon\left(6 a^{2}+h^{2}-2 \epsilon^{2}\right)\left(a^{2} M_{1}+M_{3}\right)\right. \\
& \left.+2\left(6 a^{2}-h^{2}+\epsilon^{2}\right) M_{4}\right]
\end{aligned}
$$

where for the sake of conciseness we have omitted higher powers in $q$ (although we have carried out computations up to $q^{4}$ ). For the gauge theory partition function one finds

$$
Z_{\text {inst }+ \text { tree }}=q^{-\frac{a^{2}}{h^{2}}}\left(1-\frac{q}{h^{2}\left(4 a^{2}-\epsilon^{2}\right)}\left(2 a^{4}+a^{2}\left(-\epsilon M_{1}+2 M_{2}\right)-\epsilon M_{3}+2 M_{4}\right)+\ldots\right) .
$$

The explicit form of $Z_{\text {one-loop }}$ is irrelevant to our purposes since it is $\tau$ independent. It is then not hard to get convinced that (4.29) and (4.30) satisfy the chiral ring relations (4.26) to order $q$. We have checked this up to order $q^{4}$.

\subsubsection{Deformed Seiberg-Witten curve: $h=\epsilon_{1} \epsilon_{2}=0$}

Chiral relations of the type of (4.26) are believed to exist for all chiral correlators but the explicit form of these relations becomes quickly too complicated. The situation is dramatically improved in the limit $h \rightarrow 0$, where as we will show the chiral correlators can be efficiently extracted from the deformed Seiberg-Witten curve governing the dynamics of the theory. We perform the limit keeping $\left\langle\operatorname{tr} \tilde{\varphi}^{2}\right\rangle$ or equivalently $\mathcal{F}=-h^{2} \ln Z_{\text {inst }}$ finite. For $\mathrm{SU}(2)$ gauge theory with four fundamentals the deformed Seiberg-Witten curve is given by the difference equation $[25,26]$

$$
-q Q(z-\epsilon) y(z) y(z-\epsilon)+(1+q) P(z) y(z)-1=0
$$

with

$$
P(z)=z^{2}-u_{1} z+u_{2} \quad Q(z)=1+\sum_{\ell=1}^{4} M_{\ell} z^{\ell}
$$

The chiral correlators can be extracted from the expansion at large $z$ of

$$
\partial_{z} \log y(z)=\left\langle\operatorname{tr} \frac{1}{z-\tilde{\varphi}}\right\rangle=\frac{2}{z}+\left\langle\frac{\operatorname{tr} \tilde{\varphi}}{z^{2}}\right\rangle+\left\langle\frac{\operatorname{tr} \tilde{\varphi}^{2}}{z^{3}}\right\rangle+\ldots
$$

Writing

$$
y(z)=\sum_{i=2}^{\infty} y_{i} z^{-i}
$$


and using (4.33) one finds the chiral correlators as functions of the $y_{i}$ 's.

$$
\begin{aligned}
\left\langle\operatorname{tr} \frac{1}{z-\tilde{\varphi}}\right\rangle= & \frac{2}{z}+\frac{y_{3}}{z^{2}}+\frac{-y_{3}^{2}+2 y_{4}}{z^{3}}+\frac{y_{3}^{3}-3 y_{3} y_{4}+3 y_{5}}{z^{4}} \\
& +\frac{-y_{3}^{4}+4 y_{3}^{2} y_{4}-2 y_{4}^{2}-4 y_{3} y_{5}+4 y_{6}}{z^{5}}+\ldots
\end{aligned}
$$

On the other hand, plugging (4.34) into the difference equation (4.31) one can solve for $y_{i}$ order by order in $i$ in terms of $u_{1}, u_{2}$. Moreover imposing $\operatorname{tr} \tilde{\varphi}=0$ requires $y_{3}=0$ and determines $u_{1}$ while $u_{2}$ can be solved in terms of $\left\langle\operatorname{tr} \tilde{\varphi}^{2}\right\rangle$. The results are

$$
u_{1}=-\frac{q\left(M_{1}-2 \epsilon\right)}{1+q} \quad u_{2}=\frac{(-1+q)\left\langle\operatorname{tr} \tilde{\varphi}^{2}\right\rangle+2 q M_{2}+2 q \epsilon\left(\epsilon-M_{1}\right)}{2(1+q)}
$$

Using these relations one can relate all correlators to $\left\langle\operatorname{tr} \tilde{\varphi}^{2}\right\rangle$ finding equations of the chiral ring type. Explicitly

$$
\begin{aligned}
\left\langle\operatorname{tr} \tilde{\varphi}^{3}\right\rangle= & \frac{3 q}{1-q}\left(\frac{1}{2}\left\langle\operatorname{tr} \tilde{\varphi}^{2}\right\rangle M_{1}+M_{3}\right) \\
\left\langle\operatorname{tr} \tilde{\varphi}^{4}\right\rangle= & \frac{(1+q)}{2(1-q)}\left\langle\operatorname{tr} \tilde{\varphi}^{2}\right\rangle^{2}+\frac{2 q}{(1-q)^{2}}\left(M_{2}(1-q)+q M_{1}^{2}+\epsilon M_{1}\right)\left\langle\operatorname{tr} \tilde{\varphi}^{2}\right\rangle \\
& +\frac{4 q}{(1-q)^{2}}\left(M_{4}(1-q)+q M_{1} M_{3}+\epsilon M_{3}\right)
\end{aligned}
$$

Consistently, (4.37) reduces to (4.28) when $h$ is set to zero. Similar relations can be found for chiral correlators involving higher powers of the scalar field.

\section{$5 \quad$ AGT duals of minimal models}

We saw before in (3.8) that for the Wilson loop to be closed the ratio of the two parameters of the $\Omega$-background must be the ratio of two integers. These rational $\Omega$-backgrounds has been conjectured to be AGT dual of the minimal models [27-31]. In this section we collect evidence in favor of this duality. It is also possible to show that the insertion of a Wilson loop in the gauge partition function dual to the minimal model correlators is trivial since it contributes only an overall sign as a consequence of the rationality of the $\Omega$-background.

\subsection{The minimal models}

We start by reviewing the results for the basic correlators on the minimal models, with the focus on the Ising model. Let $p, q$ be positive co-prime integers. The minimal model $\mathcal{M}_{p, q}$ is characterized by its Virasoro central charge

$$
c=1-\frac{6(p-q)^{2}}{p q} .
$$

There are $(p-1)(q-1) / 2$ primary fields denoted as $\phi_{m, n}, m \in\{1,2, \ldots, p-1\}, n \in$ $\{1,2, \ldots, q-1\}$ with conformal dimensions

$$
\Delta_{m, n}=\alpha_{m, n}\left(Q-\alpha_{m, n}\right)
$$


where $Q=b+1 / b, b=i \sqrt{p / q}$ and

$$
\alpha_{m, n}=\frac{1-m}{2 b}+b \frac{1-n}{2}
$$

Note the identification $\phi_{m, n} \equiv \phi_{p-m, q-n}$, which reflects the symmetry of the dimension (5.2) with respect to $\alpha \leftrightarrow Q-\alpha$.

Since a primary field $\phi_{m, n}$ is degenerated at the level $m n$, any correlation function including this field satisfies a linear differential equation of order $m n$. Thus "in principle" all the correlation functions are calculable. In particular the four point functions including the field $\phi_{1,2}$ or $\phi_{2,1}$ satisfy a second order differential equation and can be explicitly expressed in terms of hypergeometric functions. An important feature of the physical 4point function in minimal models is that only a finite number of primary fields may appear in the intermediate channels, so the correlator is given by a sum (rather than an integral) of squares of the corresponding conformal blocks.

For the sake of exemplification, here and in the following, we will focus on the simplest case $(p, q)=(3,4)$ corresponding to the Ising model. In this case there are three spinless primaries

$$
o=\phi_{1,1} \equiv \phi_{2,3} \quad \sigma=\phi_{1,2} \equiv \phi_{2,2} \quad \varepsilon=\phi_{2,1} \equiv \phi_{1,3}
$$

whose dimensions are

$$
\Delta_{o}=0, \quad \Delta_{\varepsilon}=\frac{1}{16}, \quad \Delta_{\sigma}=\frac{1}{2}
$$

The non-trivial three-point structure constants are

$$
C_{o o o}=C_{\sigma \sigma o}=C_{\varepsilon \varepsilon o}=1 \quad C_{\sigma \sigma \varepsilon}=\frac{1}{2}
$$

leading to the Ising fusion rules

$$
\sigma \sigma \sim[o]+[\varepsilon] \quad \varepsilon \varepsilon \sim[o] \quad \sigma \varepsilon \sim[\sigma]
$$

The conformal blocks consistent with these fusion rules are given by (see e.g. [38])

$$
\begin{aligned}
\langle\sigma \sigma \sigma \sigma\rangle_{o} & =\frac{\sqrt{1+\sqrt{1-z}}}{\sqrt{2} z^{1 / 8}(1-z)^{1 / 8}} & \langle\sigma \sigma \sigma \sigma\rangle_{\varepsilon} & =\frac{\sqrt{2} \sqrt{1-\sqrt{1-z}}}{z^{1 / 8}(1-z)^{1 / 8}} \\
\langle\varepsilon \varepsilon \varepsilon \varepsilon\rangle_{o} & =\frac{z}{1-z}+\frac{1}{z} & \langle\sigma \varepsilon \sigma \varepsilon\rangle_{\sigma} & =\frac{1-2 z}{\sqrt{z(1-z)}} \\
\langle\varepsilon \varepsilon \sigma \sigma\rangle_{o} & =\frac{2-z}{2 z^{1 / 8} \sqrt{1-z}} & \langle\sigma \sigma \varepsilon \varepsilon\rangle_{o} & =\frac{2-z}{2 z \sqrt{1-z}}
\end{aligned}
$$

The subscripts on the l.h.s. label the operators which are exchanged. For the full correlators one finds

$$
\begin{aligned}
\langle\sigma \sigma \sigma \sigma)\rangle & =\frac{|1+\sqrt{1-z}|+|1-\sqrt{1-z}|}{2|z(1-z)|^{1 / 4}} & \langle\varepsilon \varepsilon \varepsilon \varepsilon\rangle & =\frac{\left|1-z+z^{2}\right|^{2}}{|z(1-z)|^{2}} \\
\langle\sigma \varepsilon \sigma \varepsilon\rangle & =\frac{|1-2 z|^{2}}{|z(1-z)|} & \langle\varepsilon \varepsilon \sigma \sigma\rangle & =\frac{|1-z / 2|^{2}}{|z|^{1 / 4}|1-z|}
\end{aligned}
$$




\subsection{Degenerated states vs. critical masses}

To realize the Ising model we take $(p, q)=(3,4)$ and

$$
\epsilon_{1}=\epsilon_{2}^{-1}=b=\mathrm{i} \sqrt{\frac{3}{4}}
$$

The masses, $\bar{m}_{i}$, are chosen such that the $\alpha_{i}$ 's in (4.20) belong to the set

$$
\alpha_{i} \in\left\{\alpha_{o}, \alpha_{\sigma}, \alpha_{\varepsilon}\right\}=\left\{0,-\frac{b}{2},-\frac{1}{2 b}\right\} \cup\left\{Q, Q+\frac{b}{2}, Q+\frac{1}{2 b}\right\}
$$

associated to the primary fields (5.4). Without any loss in generality we can discard insertions of the identity operator since they lead to lower point correlators. The two non-trivial possibilities for the vertex insertions at position q correspond to the choice

$$
\bar{m}_{3}+\bar{m}_{4}= \begin{cases}\epsilon_{1} & \mathcal{O}_{3}=\sigma \\ \epsilon_{2} & \mathcal{O}_{3}=\varepsilon\end{cases}
$$

with arbitrary $\bar{m}_{1}, \bar{m}_{2}$ (see (3.9) in [32]). Using (4.20) one can rewrite (5.12) as

$$
\alpha_{3}= \begin{cases}Q+\frac{b}{2} & \mathcal{O}_{3}=\sigma \\ Q+\frac{1}{2 b} & \mathcal{O}_{3}=\varepsilon\end{cases}
$$

The gauge theory partition function on $S^{4}$ is given by an integration over the vacuum expectation value $a=a_{1}=-a_{2}$. This integration can be formally evaluated with the method of the residues but the number of poles is infinite. Still, as shown in [32], at the special values of the masses (5.12) the one loop partition function vanishes and only the residue at those special values of $a$ where two single poles collide can contribute. For SU(2) this happens at two points: $a=a_{+}$or $a=a_{-}$given by

$$
a_{+}=-\bar{m}_{4} \quad \text { or } \quad a_{-}=-\bar{m}_{3}
$$

Using (4.20), (5.13), (5.14) one finds

$$
\alpha_{+}=\left\{\begin{array}{cc}
\alpha_{4}-\frac{b}{2} \\
\alpha_{4}-\frac{1}{2 b}
\end{array} \quad \alpha_{-}= \begin{cases}Q-\left(\alpha_{4}+\frac{b}{2}\right) & \mathcal{O}_{3}=\sigma \\
Q-\left(\alpha_{4}+\frac{1}{2 b}\right) & \mathcal{O}_{3}=\varepsilon\end{cases}\right.
$$

Moreover, for $a=a_{+}$, the instanton partition function receives contributions only from Young tableaux with a single row(column) at $a_{+}$and no boxes at $-a_{+}$for $\mathcal{O}_{\ni}=\sigma(\epsilon)$. Similarly, for $a=a_{-}$the relevant single-row(column) tableaux are centered at $a_{-}$. The whole instanton sum adds to a hypergeometric function [32]

$$
\begin{aligned}
& Z_{\text {inst, }+}^{\mathrm{U}(2)}={ }_{2} F_{1}\left(\begin{array}{c}
A_{1}, A_{2} \\
B
\end{array} \mid q\right) \\
& Z_{\text {inst, }-}^{\mathrm{U}(2)}={ }_{2} F_{1}\left(\begin{array}{c}
1-B+A_{1}, 1-B+A_{2} \\
2-B
\end{array} \mid q\right)
\end{aligned}
$$




\begin{tabular}{|c|c|c|c|}
\hline$\left\langle\mathcal{O}_{1} \mathcal{O}_{2} \mathcal{O}_{3} \mathcal{O}_{4}\right\rangle_{\mathcal{O}_{ \pm}}$ & \pm & $\left(\alpha_{1}, \alpha_{2}, \alpha_{3}, \alpha_{4} ; \alpha_{ \pm}\right)$ & $\left(\bar{m}_{1}, \bar{m}_{2}, \bar{m}_{3}, \bar{m}_{4} ; a_{ \pm}\right)$ \\
\hline$\langle\sigma \sigma \sigma \sigma\rangle_{\varepsilon}$ & + & $\left(-\frac{b}{2},-\frac{b}{2}, Q+\frac{b}{2},-\frac{b}{2} ;-b\right)$ & $\left(-\frac{\epsilon}{2}, \epsilon_{1}+\frac{\epsilon}{2},-\frac{\epsilon}{2}, \epsilon_{1}+\frac{\epsilon}{2} ;-\epsilon_{1}-\frac{\epsilon}{2}\right)$ \\
$\langle\sigma \sigma \sigma \sigma\rangle_{o}$ & - & $\left(-\frac{b}{2},-\frac{b}{2}, Q+\frac{b}{2},-\frac{b}{2} ; Q\right)$ & $\left(-\frac{\epsilon}{2}, \epsilon_{1}+\frac{\epsilon}{2},-\frac{\epsilon}{2}, \epsilon_{1}+\frac{\epsilon}{2} ; \frac{\epsilon}{2}\right)$ \\
$\langle\varepsilon \varepsilon \varepsilon \varepsilon\rangle_{o}$ & - & $\left(-\frac{1}{2 b},-\frac{1}{2 b}, Q+\frac{1}{2 b},-\frac{1}{2 b} ; Q\right)$ & $\left(-\frac{\epsilon}{2}, \epsilon_{2}+\frac{\epsilon}{2},-\frac{\epsilon}{2}, \epsilon_{2}+\frac{\epsilon}{2} ; \frac{\epsilon}{2}\right)$ \\
$\langle\sigma \varepsilon \sigma \varepsilon\rangle_{\sigma}$ & + & $\left(-\frac{b}{2},-\frac{1}{2 b}, Q+\frac{b}{2},-\frac{1}{2 b} ;-\frac{b}{2}-\frac{1}{2 b}\right)$ & $\left(-\epsilon_{1}, \epsilon,-\epsilon_{2}, \epsilon ;-\epsilon\right)$ \\
$\langle\sigma \sigma \varepsilon \varepsilon\rangle_{o}$ & - & $\left(-\frac{b}{2},-\frac{b}{2}, Q+\frac{1}{2 b},-\frac{1}{2 b} ; Q\right)$ & $\left(-\frac{\epsilon}{2}, \epsilon_{1}+\frac{\epsilon}{2},-\frac{\epsilon}{2}, \epsilon_{2}+\frac{\epsilon}{2} ; \frac{\epsilon}{2}\right)$ \\
$\langle\varepsilon \varepsilon \sigma \sigma\rangle_{o}$ & - & $\left(-\frac{1}{2 b},-\frac{1}{2 b}, Q+\frac{b}{2},-\frac{b}{2} ; Q\right)$ & $\left(-\frac{\epsilon}{2}, \epsilon_{2}+\frac{\epsilon}{2},-\frac{\epsilon}{2}, \epsilon_{1}+\frac{\epsilon}{2} ; \frac{\epsilon}{2}\right)$ \\
\hline
\end{tabular}

Table 1. Critical values of the masses associated to the four-point conformal blocks of the Ising model. $\mathcal{O}_{ \pm}$are the operators associated to $\alpha_{ \pm}$.

with

$$
\begin{aligned}
\mathcal{O}_{3}=\sigma: & A_{1}=\left(-\alpha_{1}+\alpha_{2}+\alpha_{+}\right) b=\left(a_{+}-\bar{m}_{1}\right) \epsilon_{1} \\
A_{2} & =\left(\alpha_{1}+\alpha_{2}+\alpha_{+}-Q\right) b=\left(a_{+}-\bar{m}_{2}\right) \epsilon_{1} \\
B & =2 \alpha_{+} b=\left(2 a_{+}+\epsilon\right) \epsilon_{1} \\
\mathcal{O}_{3}=\varepsilon: \quad A_{1} & =\frac{-\alpha_{1}+\alpha_{2}+\alpha_{+}}{b}=\frac{a_{+}-\bar{m}_{1}}{\epsilon_{1}} \\
A_{2} & =\frac{\alpha_{1}+\alpha_{2}+\alpha_{+}-Q}{b}=\frac{a_{+}-\bar{m}_{2}}{\epsilon_{1}} \\
B & =\frac{2 \alpha_{+}}{b}=\frac{2 a_{+}+\epsilon}{\epsilon_{1}}
\end{aligned}
$$

\subsubsection{The partition function and the four-points conformal blocks}

The critical values of the masses associated to the four-point conformal blocks of the Ising model are listed in table 1. Using (5.16)-(5.18) one finds the $\mathrm{U}(2)$ instanton partition functions

$$
\begin{aligned}
& Z_{\langle\sigma \sigma \sigma \sigma\rangle_{\varepsilon}}^{\mathrm{U}(2), \text { inst }}={ }_{2} F_{1}\left(\frac{3}{4}, \frac{5}{4}, \frac{3}{2}, q\right)=\frac{\sqrt{2} \sqrt{1-\sqrt{1-q}}}{\sqrt{q(1-q)}} \\
& Z_{\langle\sigma \sigma \sigma \sigma\rangle_{o}}^{\mathrm{U}(2), \text { inst }}={ }_{2} F_{1}\left(\frac{1}{4}, \frac{3}{4}, \frac{1}{2} ; q\right)=\frac{\sqrt{1+\sqrt{1-q}}}{\sqrt{2} \sqrt{1-q}} \\
& Z_{\langle\varepsilon \varepsilon \varepsilon \varepsilon\rangle_{o}}^{\mathrm{U}(2), \text { inst }}={ }_{2} F_{1}\left(-\frac{1}{3}, \frac{4}{3},-\frac{2}{3}, q\right)=\frac{1-q+q^{2}}{(1-q)^{\frac{5}{3}}} \\
& Z_{\langle\sigma \varepsilon \sigma \varepsilon\rangle_{\sigma}}^{\mathrm{U}(2), \text { inst }}={ }_{2} F_{1}\left(-1,-\frac{1}{2},-\frac{1}{4}, q\right)=(1-2 q) \\
& Z_{\langle\sigma \sigma \varepsilon \varepsilon\rangle_{o}}^{\mathrm{U}(2), \text { inst }}=Z_{\langle\varepsilon \varepsilon \sigma \sigma\rangle_{o}}^{\mathrm{U}(2), \text { inst }}={ }_{2} F_{1}\left(\frac{1}{4},-1, \frac{1}{2}, q\right)=1-\frac{1}{2} q
\end{aligned}
$$

with the subscript indicating the dual CFT correlator. The Ising conformal blocks (5.8) are reproduced from $(5.20)-(5.23)$ via $(4.22)$

$$
\left\langle\mathcal{O}_{1} \mathcal{O}_{2} \mathcal{O}_{3} \mathcal{O}_{4}\right\rangle_{\mathcal{O}_{ \pm}}=q^{\Delta_{ \pm}-\Delta_{3}-\Delta_{4}} Z_{\left\langle\mathcal{O}_{1} \mathcal{O}_{2} \mathcal{O}_{3} \mathcal{O}_{4}\right\rangle_{\mathcal{O}_{ \pm}}}^{\mathrm{U}(2), \text { inst }} \times \begin{cases}(1-q)^{\alpha_{2} b} & \mathcal{O}_{3}=\sigma \\ (1-q)^{\frac{\alpha_{2}}{b}} & \mathcal{O}_{3}=\varepsilon\end{cases}
$$


The remaining critical partition functions $Z_{\langle\varepsilon \varepsilon \varepsilon \varepsilon\rangle_{\mathcal{O}_{+}}}^{\mathrm{U}(2), i_{s t}}, Z_{\langle\sigma \varepsilon \sigma \varepsilon\rangle_{\mathcal{O}_{-}}}^{\mathrm{U}(2) \text {,int }}, Z_{\langle\sigma \sigma \varepsilon \varepsilon\rangle_{\mathcal{O}_{+}}}^{\mathrm{U}(2) \text { inst }}, Z_{\langle\varepsilon \varepsilon \sigma \sigma\rangle_{\mathcal{O}_{+}}}^{\mathrm{U}(2) \text {,inst }}$ have no counterpart in the minimal model side. Indeed, as we will see in the next section, they do not contribute to the gauge partition function on $S^{4}$ since the one-loop partition function vanishes at the corresponding critical values.

\subsubsection{The gauge partition function on $S^{4}$ vs the four-point correlator}

For the critical choice of masses (5.13) the $\mathrm{U}(2)$ gauge partition function on $S^{4}(3.1)$ reduces to $[32]$

$$
Z_{\left\langle\mathcal{O}_{1} \mathcal{O}_{2} \mathcal{O}_{3} \mathcal{O}_{4}\right\rangle}^{\mathrm{U}(2)}=C_{+}\left|q^{\Delta_{+}-\Delta_{3}-\Delta_{4}} Z_{\left\langle\mathcal{O}_{1} \mathcal{O}_{2} \mathcal{O}_{3} \mathcal{O}_{4}\right\rangle_{\mathcal{O}_{+}}}^{\mathrm{U}(2)}\right|^{2}+C_{-}\left|q^{\Delta_{-}-\Delta_{3}-\Delta_{4}} Z_{\left\langle\mathcal{O}_{1} \mathcal{O}_{2} \mathcal{O}_{3} \mathcal{O}_{4}\right\rangle_{\mathcal{O}_{-}}}^{\mathrm{U}(2)}\right|^{2}
$$

where we used (3.5) or equivalently $c=q^{\frac{Q^{2}}{4}-\Delta_{3}-\Delta_{4}}$ to rewrite $c q^{-a_{ \pm}^{2}}=q^{\Delta_{ \pm}-\Delta_{3}-\Delta_{4}}$. The constants $C_{ \pm}$are defined by

$$
C_{ \pm}=\operatorname{Res}_{a_{ \pm}}\left|Z_{\left\langle\mathcal{O}_{1} \mathcal{O}_{2} \mathcal{O}_{3} \mathcal{O}_{4}\right\rangle_{a}}^{\text {one-loop }}\right|^{2}
$$

and can be interpreted in the dual CFT as the product of the two three-point functions involved in the corresponding conformal block

$$
C_{ \pm}=C_{\mathcal{O}_{1} \mathcal{O}_{2} \mathcal{O}_{ \pm}} C_{\mathcal{O}_{ \pm} \mathcal{O}_{3} \mathcal{O}_{4}}
$$

The ratio between the two is given by [32]

$$
\frac{C_{-}}{C_{+}}=\frac{\gamma(B) \gamma(B-1)}{\gamma\left(A_{1}\right) \gamma\left(A_{2}\right) \gamma\left(B-A_{1}\right) \gamma\left(B-A_{2}\right)}
$$

with $\gamma(x)=\frac{\Gamma(x)}{\Gamma(1-x)}$. Plugging the special values of $\alpha_{i}$ from table 1 into (5.28) one finds

$$
\begin{array}{|c|ccccc|}
\hline & \langle\sigma \sigma \sigma \sigma\rangle & \langle\varepsilon \varepsilon \varepsilon \varepsilon\rangle & \langle\sigma \varepsilon \sigma \varepsilon\rangle & \langle\sigma \sigma \varepsilon \varepsilon\rangle & \langle\varepsilon \varepsilon \sigma \sigma\rangle \\
\hline C_{-} / C_{+} & 4 & \infty & 0 & \infty & \infty \\
\hline
\end{array}
$$

Adopting the natural normalization $C_{\mathcal{O O}}=1$ one gets from (5.27) the correct values of the Ising OPE structure constants (5.6). Thus one finds the gauge theory partition functions

$$
\begin{aligned}
Z_{\langle\sigma \sigma \sigma \sigma\rangle}^{\mathrm{U}(2)} & =\frac{|1+\sqrt{1-q}|+|1-\sqrt{1-q}|}{2|q|^{1 / 4}|1-q|} ; \\
Z_{\langle\varepsilon \varepsilon \varepsilon \varepsilon\rangle}^{\mathrm{U}(2)} & =\frac{\left|1-q+q^{2}\right|^{2}}{|q|^{2}|1-q|^{\frac{10}{3}}} ; \\
Z_{\langle\varepsilon \varepsilon \sigma \sigma\rangle}^{\mathrm{U}(2)} & =\frac{|1-q / 2|^{2}}{|q|^{1 / 4}} \\
Z_{\langle\sigma \varepsilon \sigma \varepsilon\rangle}^{\mathrm{U}(2)} & =\frac{|1-2 q|^{2}}{|q|}
\end{aligned}
$$

Notice that all formulae in the right hand side of (5.30) are well defined in the entire complex plane. In particular, going around $q=1$ the two terms in $Z_{\langle\sigma \sigma \sigma \sigma\rangle}^{\mathrm{U}(2)}$ get exchanged, so the relative coefficient $C_{-} / C_{+}=4$ is crucial to ensure the single-valueness of the correlator. 
Finally the Ising model correlators can be read from (5.30) after factoring out the U(1) contribution

$$
\left\langle\mathcal{O}_{1} \mathcal{O}_{2} \mathcal{O}_{3} \mathcal{O}_{4}\right\rangle=Z_{\left\langle\mathcal{O}_{1} \mathcal{O}_{2} \mathcal{O}_{3} \mathcal{O}_{4}\right\rangle}^{\mathrm{U}(2)} \times \begin{cases}|1-q|^{2 \alpha_{2} b} & \mathcal{O}_{3}=\sigma \\ |1-q|^{\frac{2 \alpha_{2}}{b}} & \mathcal{O}_{3}=\varepsilon\end{cases}
$$

with results in perfect agreement with (5.9).

\section{Acknowledgments}

The authors want to thank G.Bonelli and A.Tanzini for many interesting discussions. The research of R.P. is partially supported by a Visiting Professor Fellowship from the University of Roma Tor Vergata, by the Volkswagen foundation of Germany, by a grant of the Armenian State Council of Science 13-1C278 and by the Armenian-Russian grant "Common projects in Fundamental Scientific Research"-2013.

Open Access. This article is distributed under the terms of the Creative Commons Attribution License (CC-BY 4.0), which permits any use, distribution and reproduction in any medium, provided the original author(s) and source are credited.

\section{References}

[1] N. Drukker, D.J. Gross and H. Ooguri, Wilson loops and minimal surfaces, Phys. Rev. D 60 (1999) 125006 [hep-th/9904191] [INSPIRE].

[2] K. Zarembo, Supersymmetric Wilson loops, Nucl. Phys. B 643 (2002) 157 [hep-th/0205160] [INSPIRE].

[3] N. Drukker, S. Giombi, R. Ricci and D. Trancanelli, Supersymmetric Wilson loops on $S^{3}$, JHEP 05 (2008) 017 [arXiv: 0711.3226] [INSPIRE].

[4] J.K. Erickson, G.W. Semenoff and K. Zarembo, Wilson loops in $N=4$ supersymmetric Yang-Mills theory, Nucl. Phys. B 582 (2000) 155 [hep-th/0003055] [INSPIRE].

[5] E. Brézin, C. Itzykson, G. Parisi and J.B. Zuber, Planar Diagrams, Commun. Math. Phys. 59 (1978) 35 [inSPIRE].

[6] N. Drukker and D.J. Gross, An exact prediction of $N=4$ SUSYM theory for string theory, J. Math. Phys. 42 (2001) 2896 [hep-th/0010274] [INSPIRE].

[7] V. Pestun, Localization of gauge theory on a four-sphere and supersymmetric Wilson loops, Commun. Math. Phys. 313 (2012) 71 [arXiv:0712.2824] [INSPIRE].

[8] N. Nekrasov and A. Okounkov, Seiberg-Witten theory and random partitions, hep-th/0306238 [INSPIRE].

[9] M. Billó, M. Frau, F. Fucito and A. Lerda, Instanton calculus in RR background and the topological string, JHEP 11 (2006) 012 [hep-th/0606013] [INSPIRE].

[10] N.A. Nekrasov, Seiberg-Witten prepotential from instanton counting, Adv. Theor. Math. Phys. 7 (2004) 831 [hep-th/0206161] [INSPIRE]. 
[11] R. Flume and R. Poghossian, An algorithm for the microscopic evaluation of the coefficients of the Seiberg-Witten prepotential, Int. J. Mod. Phys. A 18 (2003) 2541 [hep-th/0208176] [INSPIRE].

[12] U. Bruzzo, F. Fucito, J.F. Morales and A. Tanzini, Multiinstanton calculus and equivariant cohomology, JHEP 05 (2003) 054 [hep-th/0211108] [INSPIRE].

[13] H. Nakajima and K. Yoshioka, Lectures on instanton counting, math/0311058 [INSPIRE].

[14] A.S. Losev, A. Marshakov and N.A. Nekrasov, Small instantons, little strings and free fermions, hep-th/0302191 [INSPIRE].

[15] N. Hama and K. Hosomichi, Seiberg-Witten Theories on Ellipsoids, JHEP 09 (2012) 033 [arXiv: 1206.6359] [INSPIRE].

[16] D. Gaiotto, $N=2$ dualities, JHEP 08 (2012) 034 [arXiv:0904.2715] [INSPIRE].

[17] L.F. Alday, D. Gaiotto and Y. Tachikawa, Liouville Correlation Functions from Four-dimensional Gauge Theories, Lett. Math. Phys. 91 (2010) 167 [arXiv:0906.3219] [INSPIRE].

[18] L.F. Alday, D. Gaiotto, S. Gukov, Y. Tachikawa and H. Verlinde, Loop and surface operators in $N=2$ gauge theory and Liouville modular geometry, JHEP 01 (2010) 113 [arXiv:0909.0945] [INSPIRE].

[19] N. Drukker, J. Gomis, T. Okuda and J. Teschner, Gauge Theory Loop Operators and Liouville Theory, JHEP 02 (2010) 057 [arXiv:0909.1105] [INSPIRE].

[20] G.W. Semenoff and K. Zarembo, More exact predictions of SUSYM for string theory, Nucl. Phys. B 616 (2001) 34 [hep-th/0106015] [INSPIRE].

[21] N.A. Nekrasov and S.L. Shatashvili, Quantization of Integrable Systems and Four Dimensional Gauge Theories, arXiv:0908.4052 [INSPIRE].

[22] G. Bonelli and A. Tanzini, Hitchin systems, $N=2$ gauge theories and W-gravity, Phys. Lett. B 691 (2010) 111 [arXiv:0909.4031] [INSPIRE].

[23] G. Bonelli, A. Sciarappa, A. Tanzini and P. Vasko, Six-dimensional supersymmetric gauge theories, quantum cohomology of instanton moduli spaces and $g l(N)$ Quantum Intermediate Long Wave Hydrodynamics, JHEP 07 (2014) 141 [arXiv: 1403.6454] [INSPIRE].

[24] F. Cachazo, M.R. Douglas, N. Seiberg and E. Witten, Chiral rings and anomalies in supersymmetric gauge theory, JHEP 12 (2002) 071 [hep-th/0211170] [INSPIRE].

[25] R. Poghossian, Deforming SW curve, JHEP 04 (2011) 033 [arXiv:1006.4822] [INSPIRE].

[26] F. Fucito, J.F. Morales, D.R. Pacifici and R. Poghossian, Gauge theories on $\Omega$-backgrounds from non commutative Seiberg-Witten curves, JHEP 05 (2011) 098 [arXiv:1103.4495] [INSPIRE].

[27] R. Santachiara and A. Tanzini, Moore-Read Fractional Quantum Hall wavefunctions and SU(2) quiver gauge theories, Phys. Rev. D 82 (2010) 126006 [arXiv:1002.5017] [INSPIRE].

[28] B. Estienne, V. Pasquier, R. Santachiara and D. Serban, Conformal blocks in Virasoro and W theories: Duality and the Calogero-Sutherland model, Nucl. Phys. B 860 (2012) 377 [arXiv:1110.1101] [INSPIRE].

[29] M. Bershtein and O. Foda, AGT, Burge pairs and minimal models, JHEP 06 (2014) 177 [arXiv: 1404.7075] [INSPIRE]. 
[30] K.B. Alkalaev and V.A. Belavin, Conformal blocks of $W_{N}$ minimal models and AGT correspondence, JHEP 07 (2014) 024 [arXiv: 1404.7094] [INSPIRE].

[31] V. Belavin, O. Foda and R. Santachiara, AGT, N-Burge partitions and $\mathcal{W}_{N}$ minimal models, JHEP 10 (2015) 073 [arXiv: 1507.03540] [INSPIRE].

[32] F. Fucito, J.F. Morales, R. Poghossian and D. Ricci Pacifici, Exact results in $\mathcal{N}=2$ gauge theories, JHEP 10 (2013) 178 [arXiv:1307.6612] [INSPIRE].

[33] R. Flume, F. Fucito, J.F. Morales and R. Poghossian, Matone's relation in the presence of gravitational couplings, JHEP 04 (2004) 008 [hep-th/0403057] [INSPIRE].

[34] E. D'Hoker, J. Estes and M. Gutperle, Gravity duals of half-BPS Wilson loops, JHEP 06 (2007) 063 [arXiv:0705.1004] [INSPIRE].

[35] V.A. Alba, V.A. Fateev, A.V. Litvinov and G.M. Tarnopolskiy, On combinatorial expansion of the conformal blocks arising from AGT conjecture, Lett. Math. Phys. 98 (2011) 33 [arXiv: 1012.1312] [INSPIRE].

[36] A.B. Zamolodchikov and A.B. Zamolodchikov, Structure constants and conformal bootstrap in Liouville field theory, Nucl. Phys. B 477 (1996) 577 [hep-th/9506136] [INSPIRE].

[37] H. Dorn and H.J. Otto, Two and three point functions in Liouville theory, Nucl. Phys. B 429 (1994) 375 [hep-th/9403141] [INSPIRE].

[38] L. Alvarez-Gaume, G. Sierra and C. Gomez, Topics in conformal field theory, CERN-TH-5540-89 (1989). 